\title{
Espaces verts comme une alternative de conservation de la biodiversité en villes : le cas des fourmis (Hyménoptère : Formicidae) dans le district d'Abidjan (Côte d'Ivoire)
}

Lombart M. Maurice KOUAKOU1 ; Kolo YEO ${ }^{1}$; Mouhamadou KONE ${ }^{2}$; Kaly OUATTARA ${ }^{1}$; Aymard K. KOUAKOU1 ; Thibaut DELSINNE ${ }^{3}$, Wouter DEKONINCK 4

1 Université Nangui Abrogoua, Station d'écologie de Lamto, BP 28 N'Douci, Côte d'lvoire ;

2 Université Pelefero Gon Coulibaly de Korhogo, Bp 1328 Korhogo, Côte d'Ivoire ;

${ }^{3}$ Société d'Histoire Naturelle Alcide-d'Orbigny, 57 rue de Gergovie, 63170 Aubière, France ;

${ }^{4}$ Institut Royal des Sciences Naturelles de Belgique, Rue Vautier 29, B-1000 Bruxelles, Belgique

Email : *Auteur correspondant : kouakoulombart.sn@univ-na.ci / (+225 07943844)

Original submitted in on $8^{\text {th }}$ August 2018. Published online at www.m.elewa.org on $30^{\text {th }}$ November 2018 https://dx.doi.org/10.4314/jab.v131i1.10

\section{RÉSUMÉ}

Objectif: Cette étude vise à évaluer le rôle des espaces verts dans la préservation de la biodiversité dans le district d'Abidjan (Côte d'Ivoire). Les fourmis ont été utilisées comme des indicateurs biologiques afin d'examiner l'influence des activités anthropiques sur la biodiversité de ces habitats localisés dans la matrice urbaine.

Méthodologie et résultats : Les fourmis ont été collectées à l'aide d'appât de thon et du protocole ALL (pièges fosses et Winkler) dans trois types d'espaces verts (espaces verts publics, jardins botaniques et le Parc National du Banco). Les résultats indiquent que les espaces verts sont pollués (pourcentage de nuisance compris entre 1,7\% et 28,8\%) et illégalement occupés (pourcentage d'occupation compris entre $1,08 \%$ à 52,3\%) par les activités humaines. Ces milieux abritent toutefois une faune de fourmis riche avec 176 espèces collectées. De plus, les jardins botaniques et les espaces verts publics partagent $8,51 \%$ et $42,55 \%$ des espèces de fourmis avec le Parc National du Banco, malgré leur faible connectivité à cet habitat naturel.

Conclusion et application : Cette étude suggère que les espaces verts du district d'Abidjan présentent des atouts de conservation de la biodiversité, en particulier les fourmis. Toutefois, l'occupation de ces milieux par les activités commerciales conduit à leur dégradation progressive et à la disparition des communautés animales et végétales. Les résultats de cette étude devraient interpeller les décideurs à définir une politique de gestion des espaces verts et parcs urbains afin de rehausser leur valeur de conservation de la biodiversité en ville.

Mots clés : Espaces verts urbains, conservation, biodiversité, fourmis, Abidjan, Côte d'Ivoire. 
Kouakou et al., J. Appl. Biosci. 2018 Espaces verts comme une alternative de conservation de la biodiversité en villes : le cas des fourmis (Hyménoptère : Formicidae) dans Abidjan, Côte d'Ivoire

Green areas as an alternative of biodiversity conservation in cities: the case of ants (Hymenoptera: Formicidae) in the district of Abidjan (Côte d'Ivoire)

\section{ABSTRACT}

Objective: This study aims to assess the role of green areas to preserve biodiversity in the district of Abidjan (Côte d'Ivoire). Ants were used as biological indicators in order to examine the influence of anthropogenic activities on biodiversity of these habitats located in urban matrix.

Methodology and results: Ants were sampled using tuna bait, ALL Protocol (Pitfalls and Winklers) in three types of green areas (Public green areas, Botanical Gardens and Banco National Park) under two categories of anthropogenic disturbance. Results indicate a strong pollution rate (between $1.7 \%$ and 28.8 $\%$ ) and a high percentage of illegal occupation (between $1.08 \%$ and $52.3 \%$ ).. These areas shelter a rich ant fauna with 176 species collected. In addition, Botanical garden and public green areas share $8.51 \%$ to $42.55 \%$ of ant species with Banco National Park, although they are not strongly connected.

Conclusion and application: This study suggests that the green areas of the Abidjan district have biodiversity conservation assets, especially for ants. However, the illegal occupation of these areas by commercial activities leads to their progressive degradation and the disappearance of animal and plant communities. The results of this study should challenge decision-makers to define a management policy for green areas and urban parks in order to enhance their conservation value of biodiversity in city.

Keywords: Urban green areas, conservation, biodiversity, ants, Abidjan, Côte d'Ivoire.

\section{INTRODUCTION}

La croissance rapide de la population urbaine a entrainé une expansion accélérée des écosystèmes urbains à la surface du globe (Anderson et al., 2013). Ces habitats contribuent à toutes les causes majeures de dégradation des habitats naturels car ils sont localisés dans les zones très riches en biodiversité (Luniak, 1994; Chapin III et al., 2000 ; Marzluff, 2005; McKinney, 2006). En effet, le développement urbain induit l'altération des processus biophysiques et conduit à la perte et la fragmentation des milieux naturels. Ces transformations entrainent l'extinction des espèces natives et affectent profondément la capacité des écosystèmes à fournir les services indispensables à la survie et au bien-être de l'homme (Alberti, 2010). Malgré cet effet délétère de l'urbanisation, les villes sont actuellement reconnues pour abriter une part importante de la biodiversité à l'échelle locale (Kühn et al., 2004; Kowarik, 2011; McDonnell \& Hahs, 2013). La conservation de la biodiversité dans ces habitats devient donc indispensable et constitue un enjeu important pour des perspectives sociales et de développement durable. Dans les villes, les espaces verts et parcs urbains localisés dans la matrice urbaine se révèlent être d'importants répertoires de la biodiversité locale (Goddard et al., 2010; Ferland, 2015). Ces milieux, représentés par des fragments de forêts naturelles, des jardins botaniques, des espaces publics boisés, des cimetières, des terrains de golf ou des lots vacants peuvent servir de refuge à de nombreuses espèces natives de plantes et d'animaux (Müller, 2010; Kark et al., 2006; Mata et al., 2017). De plus, mis à part leur fonction esthétique, les espaces verts et parc urbains améliorent la qualité de vie sociale et économique de la population urbaine en servant de support à de nombreux services écosystémiques tels que l'approvisionnement, la régulation et les services culturels (Jennings et al., 2016 ; Grunewald et al., 2017). Malgré ces nombreux atouts, l'intégrité des espaces verts et parcs urbains est menacée par l'utilisation compétitive des terres et des pressions dues aux activités humaines dans le paysage urbain (Abdourahamane et al., 2016). En Côte d'Ivoire, le district d'Abidjan n'échappe pas à ce phénomène de dégradation des espaces verts (Nassa, 2009; Djibril et al., 2012). Alors que, très peu d'études ont documenté le devenir des espèces végétales et animales qu'on y rencontre, et ce, bien que Nassa (2009) et Yeo et al. (2013) aient signalé les 
nombreuses menaces qui pèsent sur l'intégrité de ces milieux. Dans un tel contexte, il est indispensable d'évaluer la capacité des espaces verts et parcs urbains à servir de refuge pour les espèces animales et végétales afin d'attirer l'attention des politiques sur l'importance de ces milieux dans l'habitat urbain. Toutefois la particularité des écosystèmes urbains nécessite une approche basée sur la considération de taxa cibles dits indicateurs biologiques (Oliver \& Beattie, 1996; Lawton et al., 1998). Ces derniers sont des organismes dont les exigences écologiques sont directement affectées par les variations biotiques et abiotiques intervenant dans leur milieu de vie (Ramade, 1993). Plusieurs travaux indiquent que les fourmis sont de bons indicateurs biologiques (Yeo et al., 2011; Kone et al., 2014 ; Jamison et al., 2016). Elles constituent une composante majeure de la faune du sol dans de nombreux écosystèmes et sont considérées comme

\section{MATERIEL ET METHODES}

Description des sites d'études: L'étude a été réalisée dans le district d'Abidjan dans trois types d'espaces verts à savoir les jardins botaniques, les espaces verts publics et le parc urbain naturel du Banco considéré dans cette étude comme un milieu naturel de référence (Figure 1). Le Parc National du Banco (PNB) est un massif forestier de 3474 hectares situé à l'intérieur d'Abidjan, entre les communes de Yopougon au sud-ouest, d'Attécoubé au sud, d'Adjamé au sud-est et d'Abobo au nord-est. L'échantillonnage a été effectué au centre du parc, soit à environ 2 à $3 \mathrm{~km}$ de sa lisière.

Les jardins botaniques explorés sont le Centre National de Floristique (CNF) et le Jardin Botanique de Bingerville (JBB). Le CNF couvre une superficie de ingénieurs de l'écosystème car elles peuvent influencer la disponibilité des ressources pour les organismes vivant dans leur environnement ( Del Toro et al., 2012). Elles jouent des rôles importants dans les réseaux trophiques comme herbivores, prédateurs et mutualistes (Hölldobler \& Wilson, 1990; Stadler \& Dixon, 2005). Elles assurent également la dispersion de nombreuses espèces végétales (Kaspari, 1996) et leur sensibilité aux perturbations du milieu font d'elles des insectes cibles parfaitement adaptés à l'évaluation de l'intégrité de la diversité biologique (Yeo et al., 2013; Bharti et al., 2016).Cette étude vise à évaluer le rôle de conservation de la biodiversité des espaces verts et parcs urbains en utilisant les fourmis comme indicateur biologique. L'influence des pressions humaines a également été estimée afin d'évaluer le niveau de dégradation de ces milieux dans le district d'Abidjan et de mesurer leur impact sur les assemblages de fourmis.

13 hectares. II est situé au sein de l'Université Félix Houphouët Boigny de Cocody, entre l'ancienne route de Bingerville au nord, le quartier de la Riviera Golf au sud, le quartier de la Riviera africaine à l'est et l'Unité de Formation et de Recherche (UFR) des Sciences économiques et de gestion à l'ouest. II est constitué d'un arboretum de 5 hectares caractérisé principalement par une litière abondante et un sousbois dégagé. On y trouve également une jachère de 6 hectares constituée d'une forêt secondaire bien conservée avec une litière abondante et un sous-bois touffu. Quant au jardin Botanique de Bingerville, il est situé à $18 \mathrm{~km}$ d'Abidjan dans la commune de Bingerville. II couvre une superficie de 56 hectares comprenant une forêt secondaire et une jachère. 


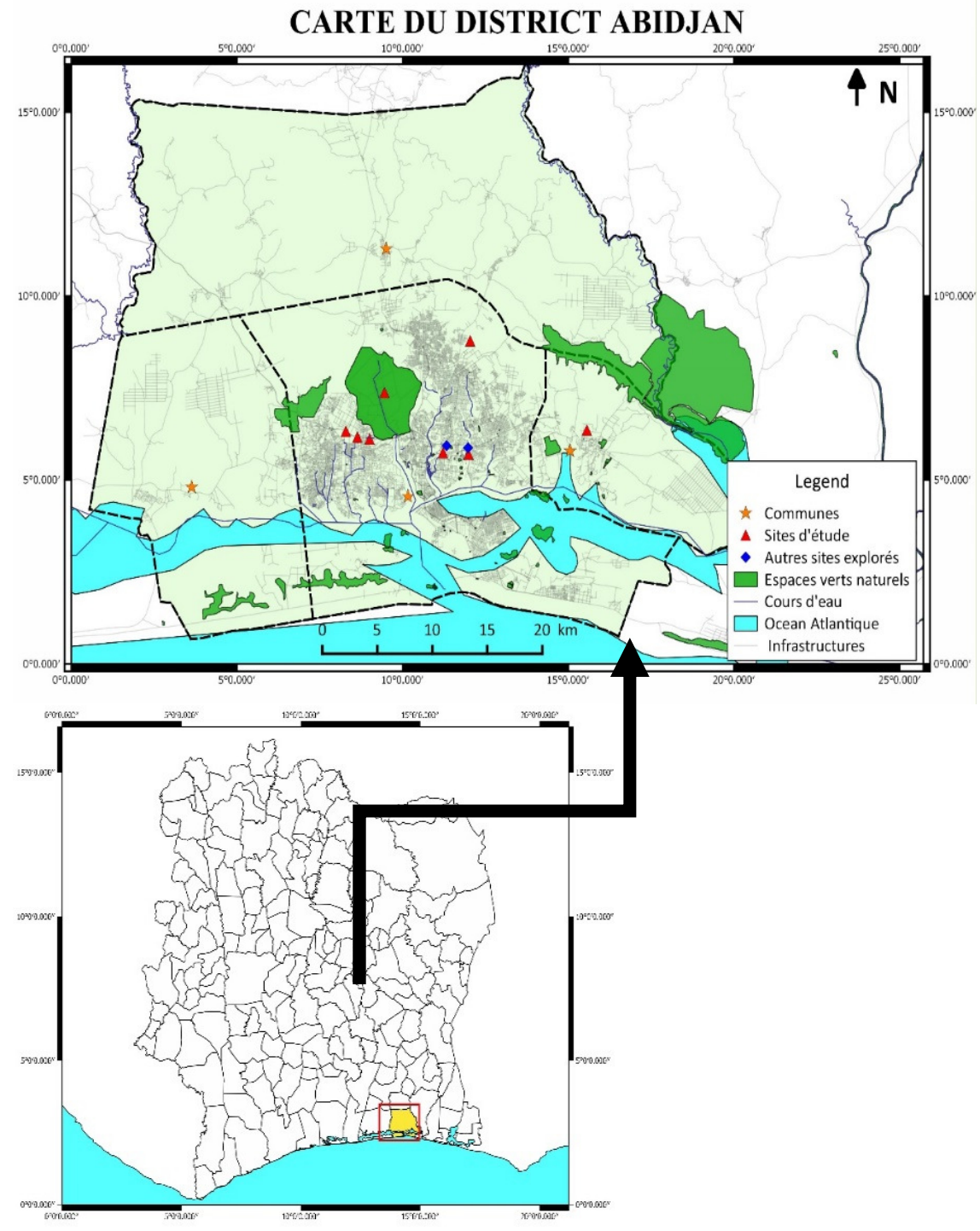

Figure 1: Localisation géographique de la zone d'étude

Les espaces verts publics sont des lieux aménagés qu'on rencontre au bas des échangeurs des ponts ou aux ronds-points des grandes voies dans le district d'Abidjan. Les espaces verts publics explorés sont celui du 1 er pont à Yopougon (EVP 1er), du $2^{\mathrm{e}}$ pont à Yopougon (EVP 2ème), du 3 ème pont à Yopougon (EVP 3 ème), du carrefour de la vie à Cocody (EVP la vie) et du Palmier à Abobo (EVP Palmier). Leur surface varie de
1,17 ha à 7,58 ha (Tableau 1). La végétation est majoritairement dominée par des arbres plantés, du gazon et des plantes horticoles.

Échantillonnage des fourmis: L'échantillonnage a été conduit en utilisant la méthode des appâts et le protocole ALL (Ants of the Leaf Litter) le long des transects de 200 mètres (soit 3 transects d'appâts et 3 transects avec le protocole ALL). 
Kouakou et al., J. Appl. Biosci. 2018 Espaces verts comme une alternative de conservation de la biodiversité en villes : le cas des fourmis (Hyménoptère : Formicidae) dans Abidjan , Côte d'Ivoire

Tableau 1 : Localisation des espaces verts et parcs urbains explorés au cours de l'étude

\begin{tabular}{lccc}
\hline Espace verts et parcs urbains & Coordonnées & $\begin{array}{c}\text { Superficie } \\
\text { en hectare }\end{array}$ & Type d'espace vert \\
\hline Parc National du Banco & $\mathrm{N}: 05^{\circ} 23^{\prime} 13.8^{\prime \prime} \mathrm{W}: 004^{\circ} 02^{\prime} 9.48^{\prime \prime}$ & 3474 & Aire protégée \\
Jardin Botanique de Bingerville & $\mathrm{N}: 05^{\circ} 21^{\prime} 9.10^{\prime \prime} \mathrm{W}: 003^{\circ} 53^{\prime} 10.14^{\prime \prime}$ & 56 & Jardin botanique \\
Centre National Floristique & $\mathrm{N}: 05^{\circ} 20^{\prime} 85.2^{\prime \prime} \mathrm{W}: 003^{\circ} 59^{\prime} 05.6^{\prime \prime}$ & 13 & Jardin botanique \\
EVP Palmier & $\mathrm{N}: 05^{\circ} 25^{\prime} 85.6^{\prime \prime} \mathrm{W}: 003^{\circ} 58^{\prime} 94.1^{\prime \prime}$ & 7,58 & Espace vert public \\
EVP La vie & $\mathrm{N}: 05^{\circ} 20^{\prime} 90.8^{\prime \prime} \mathrm{W}: 004^{\circ} 00^{\prime} 28.6^{\prime \prime}$ & 2,68 & Espace vert public \\
EVP 1er pont & $\mathrm{N}: 005^{\circ} 21^{\prime} 61.2^{\prime \prime} \mathrm{W}: 004^{\circ} 04^{\prime} 04.4^{\prime \prime}$ & 3,16 & Espace vert public \\
EVP 2ème pont & $\mathrm{N}: 05^{\circ} 21^{\prime} 57.7^{\prime \prime} \mathrm{W}: 004^{\circ} 04^{\prime} 44.5^{\prime \prime}$ & 1,17 & Espace vert public \\
EVP 3ème pont & $\mathrm{N}: 005^{\circ} 21^{\prime} 85.5^{\prime \prime} \mathrm{W}: 004^{\circ} 05^{\prime} 01.7^{\prime \prime}$ & 2,17 & Espace vert public \\
\hline
\end{tabular}

La méthode des appâts est une méthode standardisée utilisée ici pour échantillonner les fourmis des habitats dépourvus de litière. Elle a consisté à disposer des appâts de thon sur 20 points d'échantillonnage séparés de 10 mètres d'intervalle à partir du point d'origine de chaque transect. Des mies de thon ont été déposées à même le sol à l'aide d'une pince entomologique souple et marquées avec des rubans de balise. Les fourmis ont été collectées en partie toutes les 15 minutes pendant 60 minutes à partir du dépôt de l'appât.

Le protocole ALL a été appliqué uniquement au Jardin Botanique de Bingerville, au Centre National Floristique et au Parc National du Banco et a consisté à échantillonner les fourmis de la litière (Yeo, 2006). Cette méthode standardisée combine l'utilisation des sacs Winkler et les pièges-fosses (Agosti et al., 2000). Elle consiste à prélever premièrement 20 échantillons de litière dans des quadrats d'une surface de $1 \mathrm{~m}^{2}$ séparés de 10 mètres les uns des autres. Pour chacun des quadrats, la litière collectée est tamisée sur place à l'aide d'un tamis de mailles $1 \mathrm{~cm}$ afin de réduire la litière à sa partie la plus fine, mais aussi d'y concentrer la faune. De retour au laboratoire, la litière fine est versée dans des filets qui sont suspendus dans des mini extracteurs Winkler pendant 48 heures ( Yeo et al., 2013). Sur le même transect, 20 pièges fosses (verres en plastique) rempli au quart de leur volume avec un mélange d'éthanol et de glycérine sont placés dans le sol à un mètre des quadrats afin de capturer les fourmis fourrageuses qui n'ont pas été capturées par les winklers.

Évaluations des pressions anthropiques dans les espaces verts : Les pressions dans les espaces verts ont été estimées en considérant la présence ou l'absence d'occupation illégale (commerces, les garages mécaniques, les gares de transport, les baraques et les parcelles de fleuristes) de nuisances (dépôts d'ordures ménagères, d'émonde de gazon, les sites de défécation et urinoirs, les sites de brûlage de pneus). De plus, la connectivité des espaces verts a été estimée en mesurant la distance qui sépare le Parc National du Banco des autres espaces verts. Identification des fourmis : Les spécimens collectés ont été d'abord déterminés jusqu'au niveau des genres en utilisant le guide d'identification des fourmis de Bolton (1994). Ensuite, les clés de détermination de Bolton $(1976,1980)$ et la collection de référence du Docteur Yeo Kolo (Yeo, 2006), à la Station d'Écologie de Lamto, ont été utilisées pour la détermination à l'espèce. Les fourmis ont ensuite été comparées à celles de la collection de l'Institut Royal des Sciences Naturelles de Belgique (IRSNB) pour confirmer les identifications. La détermination du statut des espèces de fourmis potentiellement invasives s'est faite sur la base des travaux de Wetterer (2015); McGlynn (1999) et Holway et al. (2002).

\section{Méthode d'analyse des données}

Analyse des données sur les fourmis: Les échantillons issus de chaque méthode ont été combinés pour l'analyse statistique. A cet effet, les données d'occurrences (absence/présence) des espèces ont été utilisées plutôt que le nombre absolu d'individus des espèces (Koné, 2013 ; Yeo, 2006). Les paramètres de communautés considérés ici sont: l'abondance définie comme le nombre total des occurrences de chaque espèce dans les échantillons ; la richesse spécifique définie par le comptage du nombre d'espèces observées dans les échantillons et la diversité alpha et beta qui expriment la diversité intra et inter-habitat. La diversité alpha a été mesurée en calculant l'indice de diversité de Shannon et l'équitabilité. L'efficacité de l'échantillonnage a été testée à l'aide du taux de couverture de l'échantillonnage et les courbes d'accumulation des espèces. Le taux de couverture de l'échantillonnage est 

Kouakou et al., J. Appl. Biosci. 2018 Espaces verts comme une alternative de conservation de la
biodiversité en villes : le cas des fourmis (Hyménoptère : Formicidae) dans Abidjan, Côte d'lvoire

le ratio du nombre d'espèces observées et de la richesse spécifique estimée calculée à l'aide de l'estimateur non paramétrique Chao 2 en utilisant le logiciel Estimates 9.1. Les courbes d'accumulation des espèces sont des représentations graphiques indiquant l'évolution de la richesse spécifique en fonction de l'effort d'échantillonnage (nombre d'échantillons dans cette étude). Théoriquement, les espèces d'une communauté ont toutes été échantillonnées et on peut affirmer que la technique d'échantillonnage est efficace si cette courbe atteint une asymptote (Yeo, 2006). La variation de l'abondance et de la richesse spécifique des fourmis entre les espaces verts a été testée en utilisant les tests d'analyse de variance (ANOVA) et post hoc de Tukey. De plus, nous avons évalué l'influence des variables caractérisant chaque espace vert sur l'abondance et la richesse spécifique en calculant le coefficient de corrélation de Spearman. L'indice de similarité de Bray-Curtis a été utilisé pour mesurer la diversité beta et quantifier la ressemblance dans la composition spécifique des fourmis entre les espaces verts. La méthode d'ordination de positionnement multidimensionnel (NMDS) a permis de représenter d'une manière bidimensionnelle les sites

\section{RESULTATS}

Évaluation des catégories de pressions dans les espaces verts: La Figure 2 indique les différentes pressions observées dans les espaces verts selon le type d'espace vert, on note une différence significative des pressions de nuisance $(t=4,11 ; d l=7 ; p=0,0045)$ et du taux d'occupation humaine $\left(X^{2}=14,41 ; d l=7 ; p\right.$ $=0,0074$ ) dans les milieux échantillonnés (Tableaux 2 et 3). Les espaces verts publics subissent des selon leur ressemblance dans la composition spécifique. Enfin, nous avons conduit une analyse de similarité (ANOSIM) afin d'estimer le degré de similarité entre les espaces verts et parcs urbains sur la base de leur composition spécifique en fourmis. Tous les tests statistiques ont été réalisés avec le logiciel PAST version 3.11.

Analyse des données sur les espaces verts: Pour chaque type de pression mesuré dans un espace vert, la proportion a été calculée en faisant le rapport de la quantité de pression mesurée dans le milieu échantillonné $\left(\mathrm{Pr}_{\mathrm{i}}\right)$ sur le nombre total des quantités de pression mesurées dans tous les espaces verts et parcs urbains $\left(\Sigma \mathrm{Pr}_{\mathrm{i}}\right)$. La proportion finale de chaque catégorie de pression a été obtenue en calculant le pourcentage selon la formule suivante :

$$
\operatorname{Pr}_{\mathrm{EV}(\%)}=\left(\operatorname{Pr}_{\mathrm{i}} / \Sigma \operatorname{Pr}_{\mathrm{i})} \mathbf{x} 100\right.
$$

La variation des catégories de pression a ensuite été testée à l'aide du test $t$ de comparaison des moyennes et le test de Kruskal-Wallis.

pressions plus fortes suivis des jardins botaniques et du parc urbain du Banco. Les pressions d'occupation mesurées n'existent pas dans les milieux échantillonnés au Parc National du Banco et au Jardin Botanique de Bingerville et sont de faibles ampleurs au Centre National de Floristique, contrairement aux pressions de nuisances.

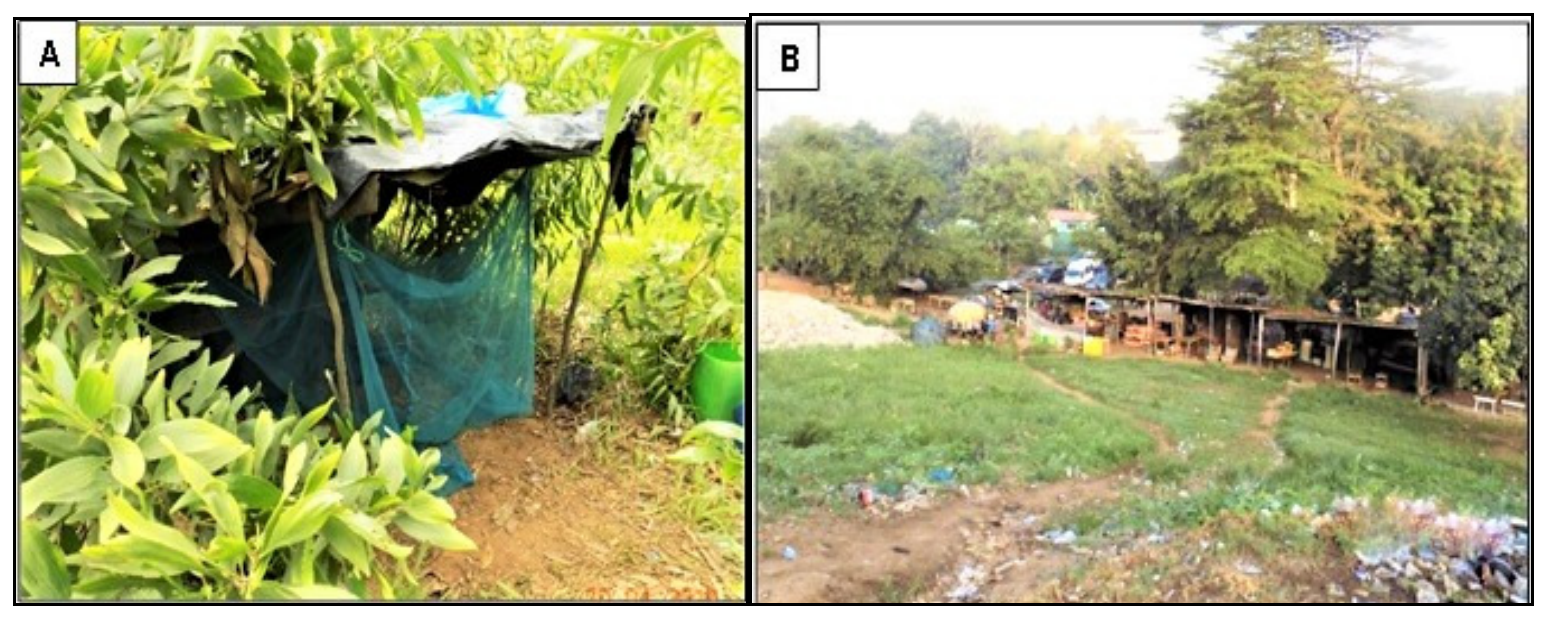


Kouakou et al., J. Appl. Biosci. 2018 Espaces verts comme une alternative de conservation de la biodiversité en villes : le cas des fourmis (Hyménoptère : Formicidae) dans Abidjan, Côte d'Ivoire

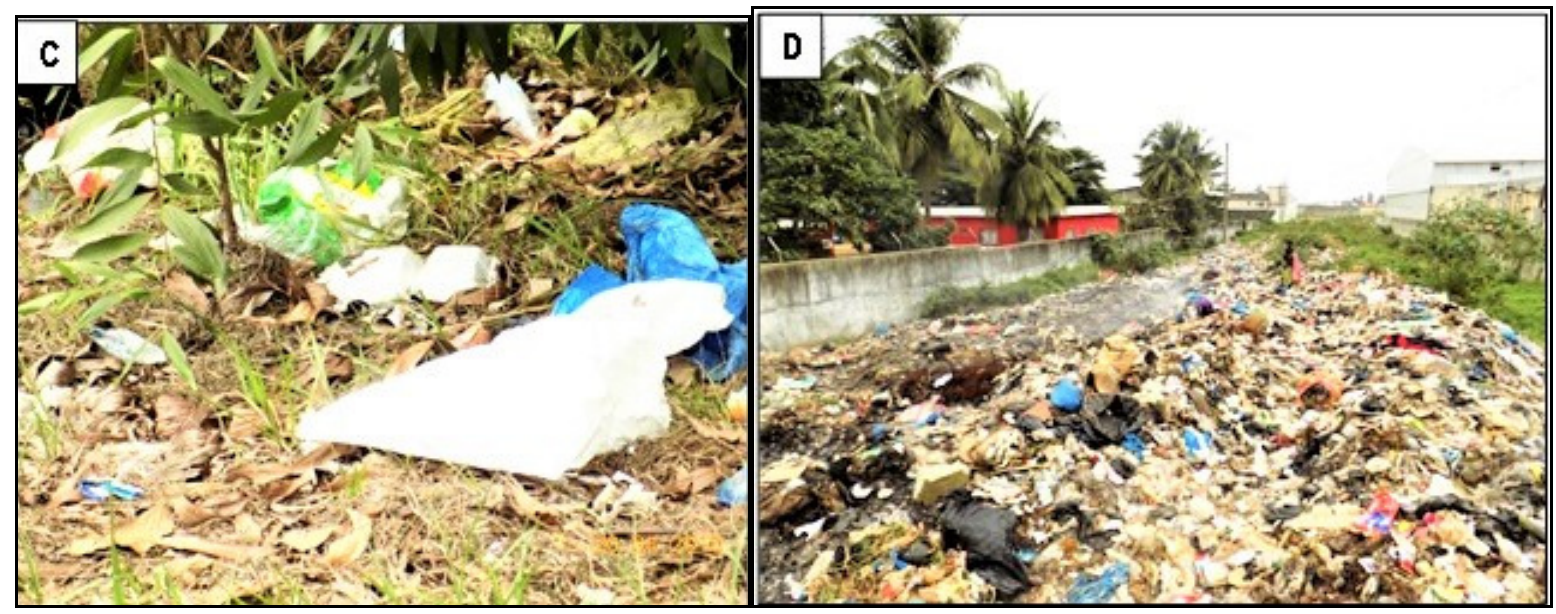

Figure 2 : Exemples d'occupations illégales ( $\mathrm{A}$ et $\mathrm{B}$ : barraques) et de pressions nuisibles dans les espaces verts ( $\mathrm{C}$ et $\mathrm{D}$ : ordures).

Tableau 2 : Pression de nuisances observées dans les espaces verts et parcs urbains

\begin{tabular}{l|l|l|l|l|l|l|l|l}
\hline Pressions de nuisance & PNB & JBB & CNF & EVP 1er & EVP 2 & EVP 3ème & $\begin{array}{l}\text { EVP } \\
\text { Palmier }\end{array}$ & EVP la vie \\
\hline $\begin{array}{l}\text { Sites de dépôt d'ordures } \\
\text { ménagères }\end{array}$ & 3 & 5 & 0 & 4 & 11 & 13 & 25 & 9 \\
Sites de dépôt d'émonde de & 0 & 7 & 3 & 19 & 3 & 18 & 10 & 4 \\
gazon & & & & & & & & \\
Sites de défécation & 0 & 6 & 2 & 0 & 3 & 4 & 6 & 2 \\
Sites urinoir & 0 & 0 & 2 & 0 & 1 & 0 & 10 & 6 \\
Brûlage de pneus & 0 & 0 & 0 & 0 & 1 & 0 & 0 & 0 \\
\hline Quantité des pressions & 3 & 18 & 7 & 23 & 19 & 35 & 51 & 21 \\
\hline Proportion (\%) & 1,7 & 10,2 & 3,9 & 13 & 10,7 & 19,8 & 28,8 & 11,8 \\
\hline
\end{tabular}

Tableau 3: Pressions d'occupation observées dans les espaces verts et parcs urbains

\begin{tabular}{|c|c|c|c|c|c|c|c|c|}
\hline Pression d'occupation & PNB & JBB & CNF & EVP 1er & EVP 2 $2^{\text {eme }}$ & EVP 3 $3^{\text {ème }}$ & EVP Palmier & EVP la vie \\
\hline Commerces & 0 & 0 & 0 & 10 & 7 & 0 & 45 & 10 \\
\hline Garages mécaniques & 0 & 0 & 0 & 0 & 2 & 0 & 2 & 6 \\
\hline Gares de transport & 0 & 0 & 0 & 0 & 0 & 0 & 0 & 3 \\
\hline Baraques & 0 & 0 & 2 & 0 & 0 & 1 & 50 & 7 \\
\hline Parcelles fleuristes & 0 & 0 & 0 & 0 & 5 & 1 & 0 & 33 \\
\hline Quantité des pressions & 0 & 0 & 2 & 10 & 14 & 2 & 97 & 59 \\
\hline Proportion (\%) & 0 & 0 & 1,08 & 5,43 & $\overline{7,61}$ & 1,08 & 52,7 & 32,06 \\
\hline
\end{tabular}

Peuplement de fourmis dans les espaces verts et parcs urbains

Diversité, richesse spécifique et abondance des fourmis : Au total 2280 échantillons ont été collectés pour un taux de couverture d'échantillonnage global de
$80,71 \%$. Les courbes d'accumulation des espèces continuent de croitre pour l'ensemble des parcs urbains et les espaces verts publics (Figure 3). Au cours de cette étude 176 espèces de fourmis ont été collectées, dont 6 potentiellement invasives (Tableau 4). Les 
fourmis identifiées appartiennent à 41 genres et sont reparties entre 8 sous-familles. Celle des Myrmicinae est la plus riche en espèces (110 espèces), tandis que celle des Apomyrminae est la plus pauvre ( 1 espèce). Les résultats ont révélé que la richesse spécifique diffèrent significativement entre les espaces verts et parcs urbains (ANOVA : $\mathrm{F}=9,08 ; d l=7 ; P<0,00001$ ). Le test post hoc de comparaison de Tukey indique que la richesse spécifique au Parc National du Banco est plus élevée que celle de l'EVP 1er, l'EVP 2ème, EVP Cocody la vie et EVP ( $p \leq 0,0005$; Tableau 5). L'indice de Shannon est également plus élevé au Parc National du Banco $(3,95)$. II est plus faible dans l'espace vert public le Palmier $(2,63)$. Par contre, l'équitabilité est plus élevée dans l'espace vert public du $3^{\mathrm{ème}}$ pont $(0,66)$ et elle est plus faible au Centre National de Floristique $(0,43)$. La comparaison de l'abondance des espèces a indiqué une distribution inégale de celle-ci dans les différentes sous-familles (ANOVA: $F=55,23$; $d l=7 ; p<0,00002$ ). Cette abondance varie également de façon significative entre les espaces verts et parcs urbains $(F=12,42 ; d l=7 ; p=0,0031)$. Les espèces de fourmis sont plus abondantes au PNB, au JBB, au CNF et dans l'espace vert public du 3ème pont. La plus faible abondance a été observée dans l'espace vert public le Palmier (Tableau 4).

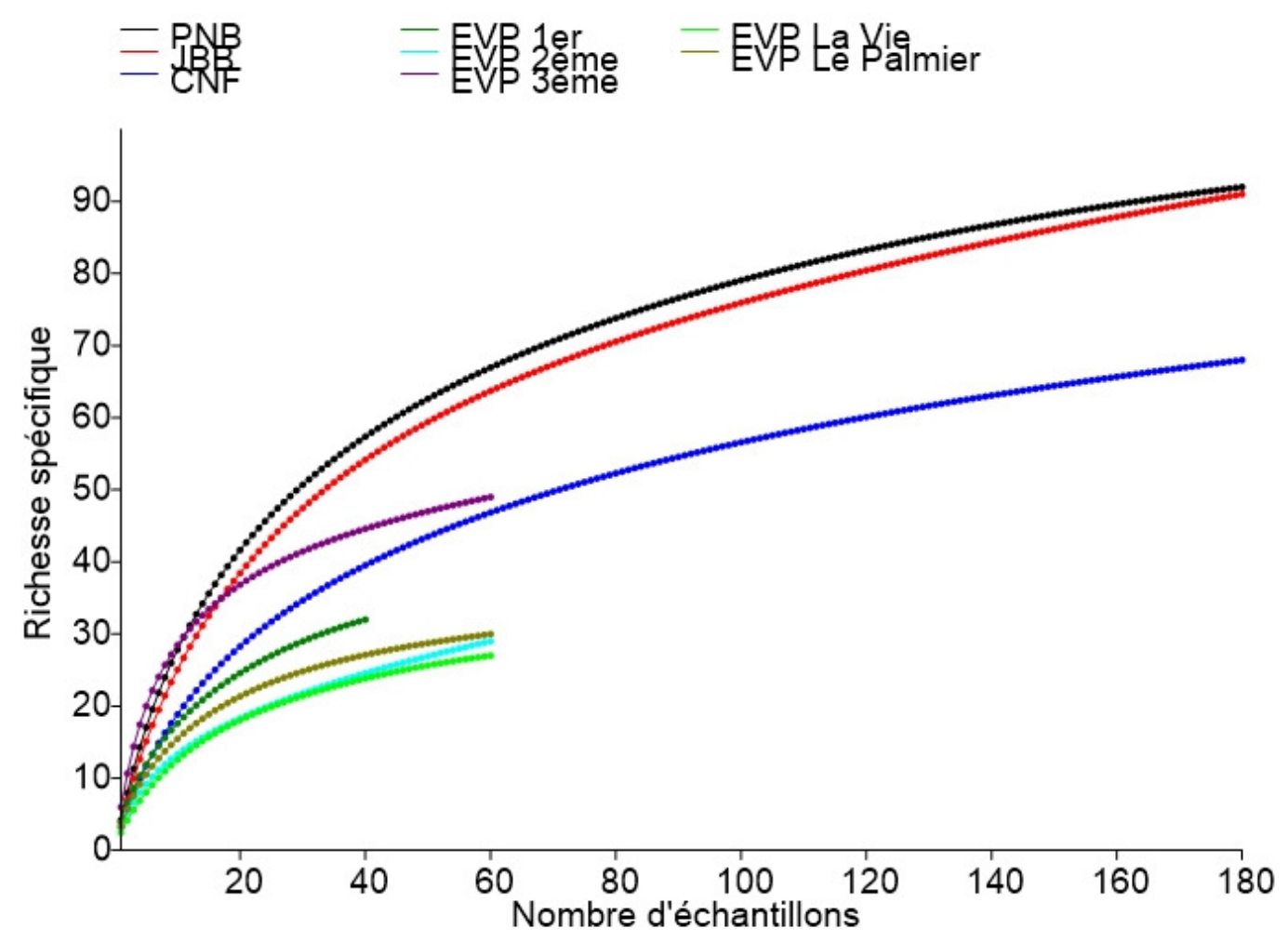

IFigure 3 : Courbes d'accumulation des espèces des espaces verts du district d'Abidjan 
Tableau 4 : Liste des espèces de fourmis collectées dans les espaces verts d'Abidjan au cours de l'étude

\begin{tabular}{|c|c|c|c|c|c|c|c|c|c|}
\hline 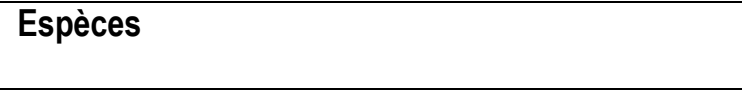 & PNB & JBB & CNF & EVP 1er & EVP 2ème & EVP 3ème & EVP Le Palmier & $\begin{array}{c}\text { EVP La } \\
\text { Vie }\end{array}$ & TOTAL \\
\hline \multicolumn{10}{|l|}{ AMBLYOPONINAE } \\
\hline Xymmer muticus (SANTSCHI, 1914) & 3 & 0 & 0 & 0 & 0 & 0 & 0 & 0 & 3 \\
\hline \multicolumn{9}{|l|}{ APOMYRMINAE } & 9 \\
\hline $\begin{array}{l}\text { Apomyrma stygia BROWN, GOTWALD \& LÉVIEUX, } \\
1971 \\
\text { DOLICHODERINAE }\end{array}$ & 0 & 1 & 0 & 0 & 0 & 0 & 0 & 0 & 1 \\
\hline Tapinoma lugubre SANTSCHI, 1917 & 1 & 12 & 19 & 20 & 11 & 24 & 8 & 23 & 118 \\
\hline Tapinoma luteum EMERY, 1895 & 0 & 0 & 0 & 0 & 0 & 1 & 0 & 0 & 1 \\
\hline $\begin{array}{l}\text { Tapinoma melanocephalum FABRICIUS, } 1793^{*} \\
\text { DORYLINAE }\end{array}$ & 1 & 9 & 0 & 14 & 0 & 6 & 0 & 3 & 33 \\
\hline Dorylus nigricans WESTWOOD, 1847 & 0 & 4 & 0 & 0 & 0 & 0 & 0 & 0 & 4 \\
\hline Dorylus sp.1 & 0 & 2 & 0 & 0 & 0 & 0 & 0 & 0 & 2 \\
\hline Dorylus sp.2 & 2 & 2 & 0 & 0 & 0 & 0 & 0 & 0 & 4 \\
\hline Dorylus sp.3 & 3 & 0 & 1 & 0 & 0 & 0 & 0 & 0 & 4 \\
\hline Dorylus sp.4 & 1 & 0 & 0 & 0 & 0 & 0 & 0 & 0 & 1 \\
\hline Lioponera sp.1 & 7 & 0 & 0 & 0 & 0 & 0 & 0 & 0 & 7 \\
\hline Parasyscia nitidulus BROWN, 1975 & 1 & 0 & 0 & 0 & 0 & 0 & 0 & 0 & 1 \\
\hline Parasyscia sp.3 & 1 & 0 & 0 & 0 & 0 & 0 & 0 & 0 & 1 \\
\hline \multicolumn{10}{|l|}{ FORMICINAE } \\
\hline Camponotus acvapimensis MAYR, 1862 & 0 & 6 & 4 & 0 & 2 & 1 & 7 & 7 & 27 \\
\hline Camponotus cinctellus GERSTÄCKER, 1859 & 0 & 13 & 10 & 0 & 0 & 16 & 2 & 9 & 50 \\
\hline Camponotus maculatus FABRICIUS, 1782 & 1 & 17 & 3 & 8 & 4 & 31 & 0 & 2 & 66 \\
\hline Camponotus shoutedeni FOREL, 1911 & 0 & 0 & 0 & 0 & 0 & 0 & 0 & 1 & 1 \\
\hline Camponotus vividus SMITH, 1858 & 0 & 2 & 1 & 1 & 0 & 2 & 0 & 2 & 8 \\
\hline Lepisiota sp.1 & 0 & 1 & 0 & 0 & 0 & 0 & 0 & 0 & 1 \\
\hline Lepisiota sp. 2 & 0 & 10 & 4 & 0 & 0 & 1 & 0 & 3 & 18 \\
\hline $\begin{array}{l}\text { Nylanderia boltoni LAPOLLA, HAWKES \& FISHER, } \\
2011\end{array}$ & 1 & 17 & 9 & 5 & 11 & 16 & 7 & 4 & 70 \\
\hline
\end{tabular}




\begin{tabular}{|c|c|c|c|c|c|c|c|c|c|}
\hline $\begin{array}{l}\text { Nylanderia brevisetula LaPolla, HAWKES \& FISHER, } \\
2011\end{array}$ & 0 & 0 & 0 & 0 & 1 & 0 & 0 & 0 & 1 \\
\hline $\begin{array}{l}\text { Nylanderia impolita LAPOLLA, HAWKES \& FISHER, } \\
2011\end{array}$ & 2 & 1 & 0 & 0 & 0 & 0 & 0 & 0 & 3 \\
\hline Nylanderia lepida (SANTSCHI, 1915) & 1 & 1 & 4 & 0 & 0 & 1 & 0 & 0 & 7 \\
\hline $\begin{array}{l}\text { Nylanderia scintilla LAPOLLA, HAWKES \& FISHER, } \\
2011\end{array}$ & 0 & 4 & 4 & 2 & 12 & 14 & 2 & 6 & 44 \\
\hline Nylanderia vividula (NYLANDER, 1846) & 0 & 0 & 0 & 0 & 0 & 0 & 1 & 0 & 1 \\
\hline Oecophylla longinoda (LATREILLE, 1802) & 3 & 7 & 25 & 1 & 3 & 6 & 1 & 7 & 53 \\
\hline Paratrechina longicornis LATREILLE, $1802^{*}$ & 0 & 10 & 49 & 3 & 2 & 9 & 5 & 14 & 92 \\
\hline Paratrechina sp.1 & 0 & 9 & 3 & 0 & 0 & 0 & 0 & 0 & 12 \\
\hline Paratrechina sp.2 & 0 & 1 & 0 & 0 & 0 & 0 & 0 & 0 & 1 \\
\hline Polyrhachis decemdentata ANDRE, 1889 & 0 & 0 & 1 & 0 & 0 & 0 & 0 & 0 & 1 \\
\hline Polyrhachis militaris FABRICIUS, 1782 & 0 & 0 & 2 & 0 & 0 & 0 & 0 & 0 & 2 \\
\hline Polyrhachis viscosa SMITH, 1858 & 0 & 0 & 1 & 0 & 0 & 0 & 0 & 0 & 1 \\
\hline \multicolumn{10}{|l|}{ MYRMICINAE } \\
\hline Baracidris meketra BOLTON, 1981 & 1 & 0 & 0 & 0 & 0 & 0 & 0 & 0 & 1 \\
\hline Cardiocondyla emeryi FOREL, $1881^{*}$ & 0 & 6 & 0 & 1 & 16 & 12 & 1 & 4 & 40 \\
\hline Cardiocondyla shuckardi FOREL, 1891 & 0 & 0 & 0 & 0 & 0 & 0 & 0 & 1 & 1 \\
\hline $\begin{array}{l}\text { Carebara lilith FISCHER, AZORSA \& HITA GARCIA, } \\
2015\end{array}$ & 0 & 1 & 1 & 0 & 0 & 0 & 0 & 0 & 2 \\
\hline Carebara distincta BOLTON \& BELSHAW, 1993 & 14 & 24 & 3 & 0 & 0 & 0 & 0 & 0 & 41 \\
\hline Carebara silvestrii SANTSCHI, 1914 & 18 & 5 & 17 & 0 & 0 & 0 & 0 & 0 & 40 \\
\hline Carebara sp.1 & 1 & 6 & 0 & 0 & 0 & 0 & 0 & 0 & 7 \\
\hline Carebara sp.2 & 2 & 0 & 0 & 0 & 0 & 0 & 0 & 0 & 2 \\
\hline Carebara sp.4 & 0 & 0 & 1 & 0 & 0 & 0 & 0 & 0 & 1 \\
\hline Carebara thoracica (WEBER, 1950) & 0 & 2 & 0 & 0 & 0 & 0 & 0 & 0 & 2 \\
\hline Cataulacus guineensis SMITH, 1853 & 0 & 0 & 1 & 0 & 0 & 0 & 0 & 0 & 1 \\
\hline Cataulacus traegaordhi SANTSCHI, 1914 & 0 & 4 & 0 & 0 & 0 & 0 & 0 & 0 & 4 \\
\hline Crematogaster sp.1 & 45 & 9 & 14 & 6 & 0 & 3 & 0 & 2 & 79 \\
\hline Crematogaster sp.2 & 2 & 4 & 0 & 0 & 0 & 0 & 0 & 0 & 6 \\
\hline Crematogaster sp. 3 & 13 & 0 & 0 & 0 & 0 & 0 & 0 & 0 & 13 \\
\hline
\end{tabular}


Kouakou et al., J. Appl. Biosci. 2018 Espaces verts comme une alternative de conservation de la biodiversité en villes : le cas des fourmis (Hyménoptère : Formicidae) dans Abidjan, Côte d'Ivoire

\begin{tabular}{|c|c|c|c|c|c|c|c|c|c|}
\hline Crematogaster sp.5 & 2 & 0 & 0 & 1 & 0 & 0 & 0 & 3 & 6 \\
\hline Crematogaster sp. 6 & 7 & 0 & 0 & 0 & 0 & 0 & 0 & 0 & 7 \\
\hline Crematogaster sp.7 & 2 & 0 & 0 & 0 & 0 & 0 & 0 & 0 & 2 \\
\hline Crematogaster sp. 9 & 4 & 0 & 0 & 0 & 0 & 0 & 0 & 0 & 4 \\
\hline Crematogaster sp.10 & 5 & 0 & 0 & 0 & 0 & 0 & 0 & 0 & 5 \\
\hline Crematogaster sp.11 & 3 & 0 & 0 & 0 & 0 & 0 & 0 & 0 & 3 \\
\hline Crematogaster sp.12 & 4 & 0 & 0 & 0 & 0 & 0 & 0 & 0 & 4 \\
\hline Meranoplus magrettii ANDRE, 1884 & 0 & 0 & 0 & 0 & 0 & 0 & 0 & 1 & 1 \\
\hline Monomorium bicolor EMERY, 1877 & 0 & 5 & 0 & 3 & 1 & 3 & 13 & 7 & 32 \\
\hline Monomorium dolatu BOLTON, 1987 & 0 & 2 & 1 & 2 & 0 & 0 & 0 & 0 & 5 \\
\hline Monomorium egens FOREL, 1910 & 1 & 0 & 0 & 0 & 0 & 0 & 0 & 0 & 1 \\
\hline Monomorium exiguum FOREL, 1894 & 0 & 1 & 4 & 0 & 0 & 2 & 0 & 3 & 10 \\
\hline Monomorium floricola JERDON, 1851* & 0 & 9 & 0 & 1 & 1 & 0 & 2 & 5 & 18 \\
\hline Monomorium invidium BOLTON, 1987 & 57 & 18 & 10 & 0 & 0 & 0 & 0 & 0 & 85 \\
\hline Monomorium occidentale BERNARD, 1953 & 0 & 0 & 0 & 0 & 0 & 16 & 0 & 3 & 19 \\
\hline Monomorium sp.1 & 0 & 1 & 0 & 1 & 1 & 6 & 0 & 1 & 10 \\
\hline Monomorium sp.2 & 0 & 0 & 0 & 0 & 0 & 5 & 0 & 0 & 5 \\
\hline Monomorium sp.3 & 0 & 1 & 0 & 0 & 0 & 1 & 0 & 0 & 2 \\
\hline Monomorium sp.4 & 16 & 8 & 3 & 0 & 0 & 0 & 1 & 0 & 28 \\
\hline Monomorium sp. 5 & 0 & 2 & 0 & 0 & 0 & 0 & 0 & 0 & 2 \\
\hline Monomorium sp. 6 & 0 & 1 & 0 & 0 & 0 & 0 & 0 & 0 & 1 \\
\hline Monomorium sp.7 & 0 & 0 & 0 & 0 & 0 & 5 & 0 & 0 & 5 \\
\hline Monomorium sp.8 & 0 & 0 & 0 & 0 & 0 & 3 & 0 & 0 & 3 \\
\hline Monomorium sp.9 & 0 & 0 & 0 & 0 & 0 & 2 & 0 & 0 & 2 \\
\hline Monomorium sp.10 & 0 & 0 & 0 & 0 & 0 & 3 & 0 & 0 & 3 \\
\hline Monomorium sp.11 & 0 & 0 & 0 & 0 & 0 & 1 & 0 & 0 & 1 \\
\hline Monomorium sp.12 & 0 & 0 & 0 & 0 & 0 & 1 & 0 & 0 & 1 \\
\hline Monomorium sp.13 & 33 & 22 & 17 & 0 & 0 & 1 & 0 & 0 & 73 \\
\hline Monomorium sp.14 & 0 & 1 & 2 & 0 & 0 & 1 & 0 & 0 & 4 \\
\hline Pheidole buchholzi MAYR, 1901 & 38 & 34 & 116 & 0 & 1 & 0 & 7 & 0 & 196 \\
\hline Pheidole excellens MAYR, 1862 & 0 & 7 & 1 & 2 & 0 & 16 & 46 & 4 & 76 \\
\hline
\end{tabular}


Kouakou et al., J. Appl. Biosci. 2018 Espaces verts comme une alternative de conservation de la biodiversité en villes : le cas des fourmis (Hyménoptère : Formicidae) dans Abidjan, Côte d'Ivoire

\begin{tabular}{|c|c|c|c|c|c|c|c|c|c|}
\hline Pheidole megacephala FABRICIUS, $1793^{*}$ & 0 & 3 & 0 & 6 & 51 & 18 & 10 & 26 & 114 \\
\hline Pheidole pulchella SANTSCHI, 1910 & 8 & 0 & 1 & 0 & 0 & 0 & 0 & 0 & 9 \\
\hline Pheidole sp.1 & 2 & 0 & 0 & 1 & 0 & 5 & 0 & 0 & 8 \\
\hline Pheidole sp.2 & 4 & 0 & 0 & 0 & 3 & 8 & 0 & 0 & 15 \\
\hline Pheidole sp.3 & 3 & 4 & 1 & 2 & 1 & 2 & 0 & 0 & 13 \\
\hline Pheidole sp.4 & 26 & 0 & 15 & 1 & 0 & 0 & 0 & 0 & 42 \\
\hline Pheidole sp. 5 & 0 & 0 & 0 & 0 & 1 & 0 & 0 & 0 & 1 \\
\hline Pheidole sp.6 & 0 & 0 & 0 & 0 & 1 & 0 & 0 & 1 & 2 \\
\hline Pheidole sp.7 & 0 & 7 & 1 & 0 & 0 & 0 & 0 & 1 & 9 \\
\hline Pheidole sp.8 & 13 & 10 & 6 & 0 & 1 & 0 & 0 & 0 & 30 \\
\hline Pheidole sp.9 & 19 & 0 & 3 & 0 & 0 & 0 & 0 & 0 & 22 \\
\hline Pheidole sp.10 & 2 & 8 & 0 & 5 & 4 & 7 & 0 & 0 & 26 \\
\hline Pheidole sp.11 & 0 & 1 & 0 & 0 & 0 & 0 & 0 & 3 & 4 \\
\hline Pheidole sp.12 & 8 & 1 & 0 & 0 & 0 & 0 & 0 & 0 & 9 \\
\hline Pheidole sp.13 & 1 & 0 & 0 & 0 & 0 & 0 & 0 & 0 & 1 \\
\hline Pheidole sp.15 & 35 & 22 & 29 & 8 & 14 & 11 & 0 & 0 & 119 \\
\hline Pheidole sp.16 & 7 & 0 & 0 & 0 & 1 & 8 & 0 & 0 & 16 \\
\hline Pheidole sp.17 & 0 & 0 & 0 & 1 & 1 & 7 & 0 & 0 & 9 \\
\hline Pheidole sp.18 & 3 & 0 & 2 & 1 & 3 & 4 & 0 & 0 & 13 \\
\hline Pheidole sp.19 & 0 & 6 & 1 & 0 & 0 & 0 & 6 & 14 & 27 \\
\hline Pheidole sp.20 & 12 & 3 & 4 & 33 & 22 & 24 & 1 & 36 & 135 \\
\hline Pheidole sp.21 & 2 & 0 & 0 & 0 & 0 & 3 & 0 & 0 & 5 \\
\hline Pristomyrmex orbiceps SANTSCHI, 1914 & 9 & 4 & 3 & 0 & 0 & 0 & 0 & 0 & 16 \\
\hline Solenopsis globularia SMITH, 1858 & 0 & 0 & 0 & 2 & 0 & 0 & 0 & 0 & 2 \\
\hline Strumigenys hensekta (BOLTON, 1983) & 0 & 0 & 2 & 0 & 0 & 0 & 0 & 0 & 2 \\
\hline Strumigenys impidora (BOLTON, 1983) & 0 & 0 & 1 & 0 & 0 & 0 & 0 & 0 & 1 \\
\hline Strumigenys laticeps (BROWN, 1962) & 4 & 0 & 0 & 0 & 0 & 0 & 0 & 0 & 4 \\
\hline Strumigenys ludovici FOREL, 1904 & 13 & 2 & 0 & 0 & 0 & 0 & 0 & 0 & 15 \\
\hline Strumigenys lujae FOREL, 1902 & 22 & 0 & 0 & 0 & 0 & 0 & 0 & 0 & 22 \\
\hline Strumigenys minkara (BOLTON, 1983) & 2 & 5 & 0 & 0 & 0 & 0 & 0 & 0 & 7 \\
\hline Strumigenys nimbrata BOLTON, 1983 & 14 & 0 & 0 & 0 & 0 & 0 & 0 & 0 & 14 \\
\hline
\end{tabular}




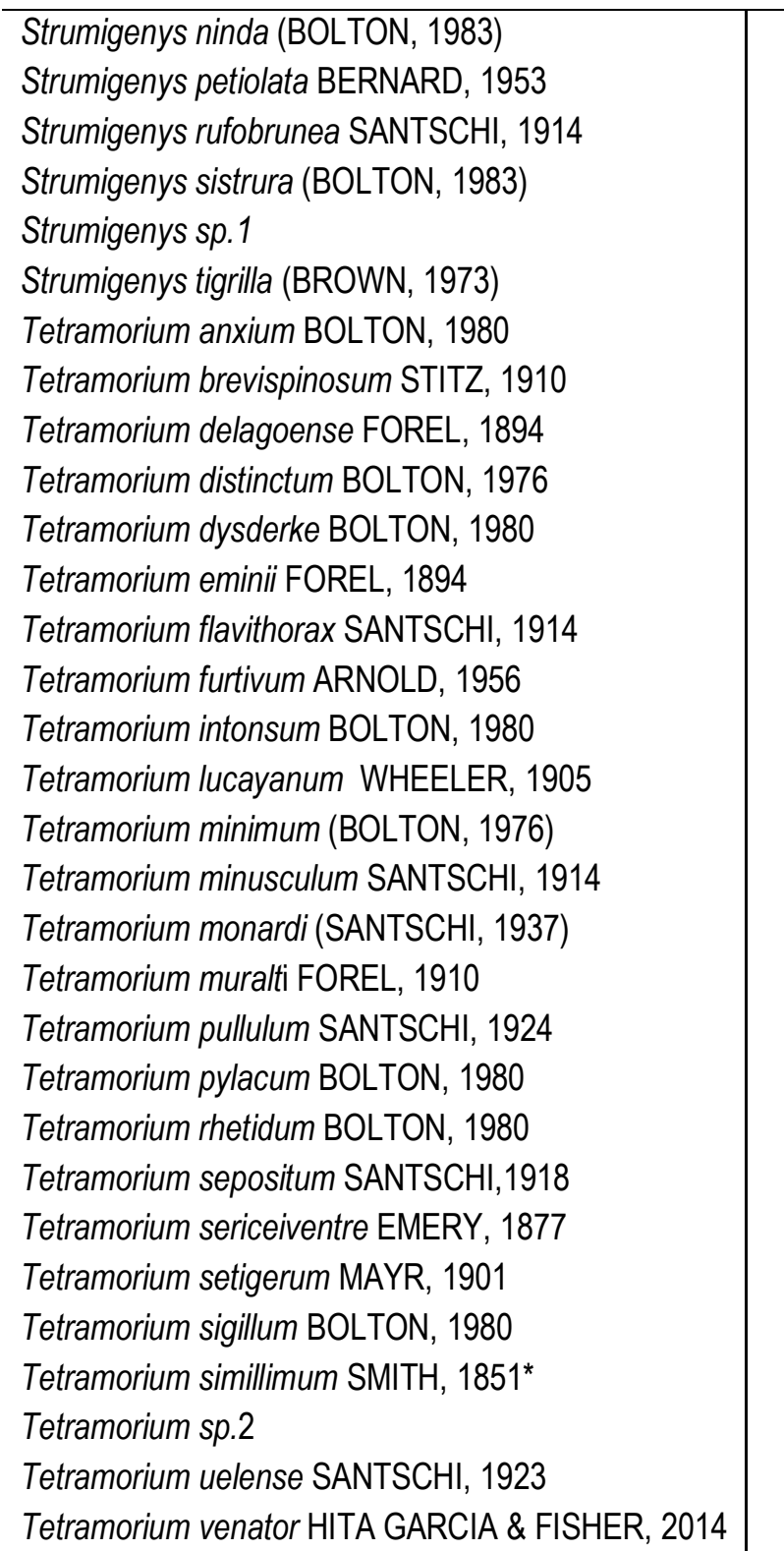

\begin{tabular}{|c|c|c|}
\hline 0 & 11 & 2 \\
\hline 16 & 5 & 5 \\
\hline 9 & 37 & 26 \\
\hline 3 & 0 & 0 \\
\hline 0 & 3 & 0 \\
\hline 9 & 0 & 0 \\
\hline 0 & 35 & 6 \\
\hline 7 & 0 & 0 \\
\hline 0 & 15 & 10 \\
\hline 0 & 1 & 0 \\
\hline 1 & 0 & 0 \\
\hline 0 & 0 & 0 \\
\hline 0 & 11 & 22 \\
\hline 7 & 2 & 0 \\
\hline 1 & 1 & 0 \\
\hline 0 & 0 & 11 \\
\hline 0 & 12 & 0 \\
\hline 0 & 0 & 1 \\
\hline 7 & 0 & 0 \\
\hline 2 & 0 & 0 \\
\hline 0 & 0 & 3 \\
\hline 2 & 0 & 1 \\
\hline 30 & 0 & 2 \\
\hline 0 & 1 & 0 \\
\hline 0 & 1 & 0 \\
\hline 0 & 1 & 0 \\
\hline 11 & 0 & 0 \\
\hline 0 & 3 & 0 \\
\hline 0 & 0 & 6 \\
\hline 0 & 1 & 0 \\
\hline 0 & 1 & 1 \\
\hline
\end{tabular}

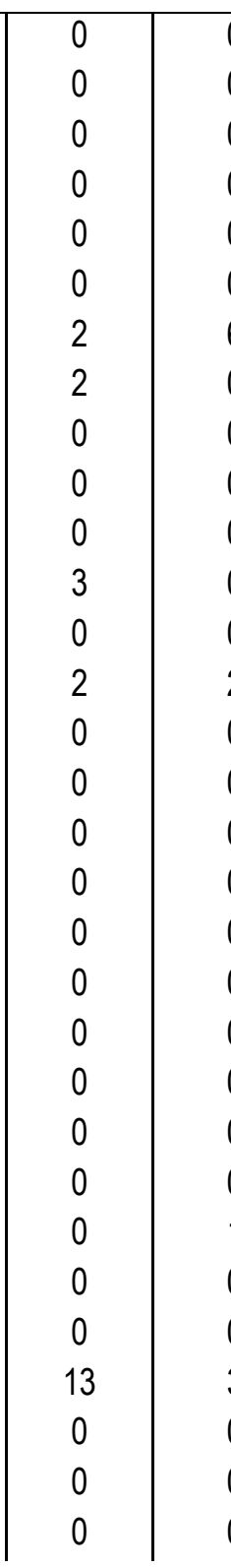

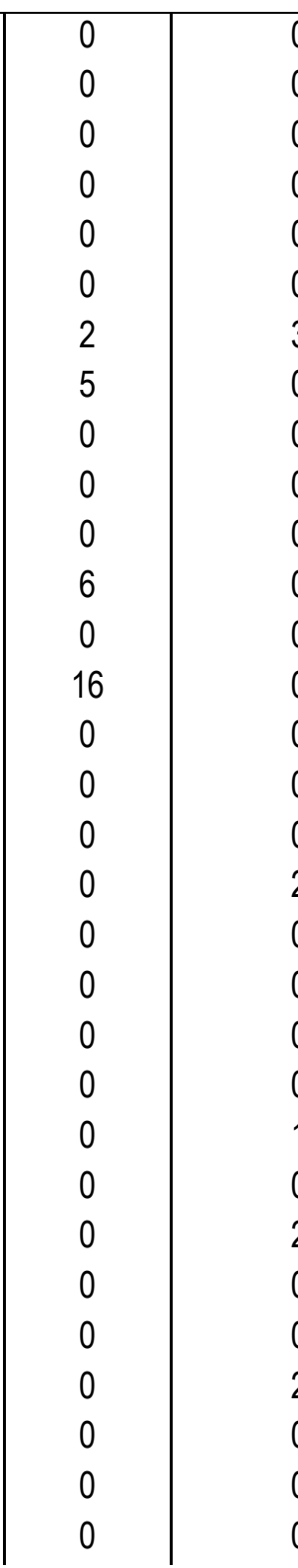

\begin{tabular}{c|c}
0 & 13 \\
0 & 26 \\
0 & 72 \\
0 & 3 \\
0 & 3 \\
0 & 9 \\
0 & 54 \\
0 & 14 \\
0 & 25 \\
0 & 1 \\
0 & 1 \\
0 & 9 \\
0 & 33 \\
0 & 29 \\
0 & 2 \\
0 & 11 \\
0 & 12 \\
1 & 4 \\
0 & 7 \\
0 & 2 \\
0 & 3 \\
0 & 3 \\
0 & 33 \\
0 & 1 \\
0 & 4 \\
0 & 1 \\
0 & 11 \\
0 & 21 \\
0 & 6 \\
0 & 1 \\
0 & 2 \\
&
\end{tabular}




\begin{tabular}{|c|c|c|c|c|c|c|c|c|c|}
\hline Tetramorium versiculum BOLTON, 1980 & 1 & 0 & 0 & 0 & 0 & 0 & 0 & 0 & 1 \\
\hline Tetramorium zapyrum BOLTON, 1980 & 0 & 0 & 0 & 0 & 0 & 0 & 1 & 0 & 1 \\
\hline \multicolumn{10}{|l|}{ PONERINAE } \\
\hline Anochetus africanus Mayr, 1865 & 3 & 1 & 5 & 0 & 0 & 0 & 0 & 0 & 9 \\
\hline Anochetus katonae FOREL, 1907 & 17 & 8 & 13 & 0 & 0 & 0 & 0 & 0 & 38 \\
\hline Anochetus siphneus BROWN, 1978 & 4 & 0 & 0 & 0 & 0 & 0 & 0 & 0 & 4 \\
\hline Asphinctopone silvestrii SANTSCHI, 1914 & 1 & 0 & 0 & 0 & 0 & 0 & 0 & 0 & 1 \\
\hline Bothroponera silvestrii SANTSCHI, 1914 & 2 & 0 & 0 & 0 & 0 & 0 & 2 & 0 & 4 \\
\hline Bothroponera soror EMERY, 1899 & 28 & 0 & 0 & 0 & 0 & 0 & 0 & 0 & 28 \\
\hline Bothroponera sp. 1 & 0 & 1 & 0 & 0 & 0 & 0 & 0 & 0 & 1 \\
\hline Bothroponera sp. 2 & 2 & 0 & 0 & 0 & 0 & 0 & 0 & 0 & 2 \\
\hline Euponera brunoi (FOREL, 1913) & 4 & 1 & 0 & 0 & 0 & 0 & 0 & 0 & 5 \\
\hline Hypoponera dulcis FOREL, 1907 & 11 & 7 & 5 & 0 & 0 & 0 & 0 & 0 & 23 \\
\hline Hypoponera molesta BOLTON \& FISHER, 2011 & 12 & 7 & 2 & 0 & 0 & 0 & 0 & 0 & 21 \\
\hline Hypoponera producta BOLTON \& FISHER, 2011 & 19 & 0 & 0 & 0 & 0 & 0 & 0 & 0 & 19 \\
\hline Hypoponera sp.2 & 1 & 0 & 0 & 0 & 0 & 0 & 0 & 0 & 1 \\
\hline Leptogenys conradti FOREL, 1913 & 1 & 1 & 0 & 0 & 0 & 0 & 0 & 0 & 2 \\
\hline Leptogenys mastax BOLTON, 1975 & 4 & 0 & 0 & 0 & 0 & 0 & 0 & 0 & 4 \\
\hline Leptogenys microps BOLTON, 1975 & 4 & 0 & 0 & 0 & 0 & 0 & 0 & 0 & 4 \\
\hline Leptogenys sp.1 & 0 & 2 & 0 & 0 & 0 & 0 & 0 & 0 & 2 \\
\hline Leptogenys sp.2 & 0 & 1 & 0 & 0 & 0 & 0 & 0 & 0 & 1 \\
\hline Leptogenys sp.3 & 1 & 0 & 0 & 0 & 0 & 0 & 0 & 0 & 1 \\
\hline Leptogenys zapyxis BOLTON, 1975 & 0 & 0 & 1 & 0 & 0 & 0 & 0 & 0 & 1 \\
\hline Loboponera sp.1 & 1 & 0 & 0 & 0 & 0 & 0 & 0 & 0 & 1 \\
\hline Mesoponera ambigua ANDRE, 1890 & 2 & 2 & 0 & 0 & 0 & 1 & 0 & 0 & 5 \\
\hline Mesoponera caffraria SMITH, 1858 & 1 & 15 & 10 & 0 & 0 & 0 & 4 & 0 & 30 \\
\hline Odontomachus assiniensis EMERY, 1892 & 0 & 0 & 0 & 0 & 0 & 1 & 0 & 0 & 1 \\
\hline Odontomachus troglodytes SANTSCHI, 1914 & 0 & 72 & 14 & 4 & 20 & 12 & 7 & 0 & 129 \\
\hline Paltothyreus tarsatus FABRICIUS, 1798 & 4 & 30 & 31 & 0 & 8 & 11 & 3 & 0 & 87 \\
\hline Plectroctena macgeei BOLTON, 1974 & 0 & 1 & 0 & 0 & 0 & 0 & 0 & 0 & 1 \\
\hline Psalidomyrmex foveolatus ANDRE, 1890 & 0 & 2 & 2 & 0 & 0 & 0 & 0 & 0 & 4 \\
\hline
\end{tabular}


Kouakou et al., J. Appl. Biosci. 2018 Espaces verts comme une alternative de conservation de la biodiversité en villes : le cas des fourmis (Hyménoptère : Formicidae) dans Abidjan, Côte d'Ivoire

\begin{tabular}{l|l|l|l|l|l|l|l|l|l}
\hline PROCERATIINAE & 8 & 0 & 1 & 0 & 0 & 0 & 0 & 0 & $\mathbf{9}$ \\
Discothyrea sp. 1 & 7 & 1 & 0 & 0 & 0 & 0 & 0 & 0 & $\mathbf{8}$ \\
Probolomyrmex guineensis TAYLOR, 1965 & 4 & 0 & 0 & 0 & 0 & 0 & 0 & 0 & $\mathbf{4}$ \\
Probolomyrmex sp.2 & 3 & 0 & 0 & 0 & 0 & 0 & 0 & 0 & $\mathbf{3}$ \\
Proceratium sp. 1 &
\end{tabular}

$\left(^{*}\right)$ Espèces de fourmis potentielles invasives 
Kouakou et al., J. Appl. Biosci. 2018 Espaces verts comme une alternative de conservation de la biodiversité en villes : le cas des fourmis (Hyménoptère : Formicidae) dans Abidjan , Côte d'Ivoire

Tableau 5 : Paramètres du peuplement de fourmis dans les espaces verts et parcs urbains

\begin{tabular}{l|c|c|c|c|c|c|c|c}
\hline Habitats & PNB & JBB & CNF & $\begin{array}{c}\text { EVP } \\
\text { 1er }\end{array}$ & $\begin{array}{c}\text { EVP } \\
\mathbf{2}^{\text {ème }}\end{array}$ & $\begin{array}{c}\text { EVP } \\
\text { 3ème }\end{array}$ & $\begin{array}{c}\text { EVP } \\
\text { La } \\
\text { vie }\end{array}$ & $\begin{array}{c}\text { EVP } \\
\text { Palmier }\end{array}$ \\
\hline Abondance (occurrences) & 764 & 671 & 580 & 157 & 209 & 360 & 191 & 147 \\
Richesse spécifique (S) & 94 & 90 & 60 & 32 & 31 & 49 & 30 & 27 \\
Richesse estimée & 104 & 121 & 93 & 37 & 33 & 58 & 35 & 30 \\
Indice de Shannon (H') & 3,95 & 3,87 & 3,37 & 2,87 & 2,70 & 3,23 & 2,85 & 2,63 \\
Equitabilité (E) & 0,55 & 0,53 & 0,43 & 0,55 & 0,49 & 0,66 & 0,57 & 0,52 \\
Couverture de l'échantillonnage & 90,3 & 74,3 & 64,5 & 86,4 & 93,9 & 84,5 & 85,7 & 90 \\
$(\%)$ & 8 & 8 & 1 & & & & 1 & \\
\hline
\end{tabular}

Influence des variables caractéristiques des espaces verts sur les paramètres des communautés de fourmis: La relation entre les variables caractéristiques des espaces verts et les paramètres de peuplement de fourmis a été évaluée en calculant une corrélation de rang de Spearman (Tableau 6). Globalement, les résultats indiquent une forte corrélation entre les pressions de nuisance et le taux d'occupation des espaces verts (Figure 4). Une corrélation négative et significative a été observée entre la richesse spécifique et l'occupation des espaces verts $(r=-0,96, p=0,0007)$, entre l'abondance des espèces et l'occupation des espaces verts $(r=-0,82, p=0,018)$ et entre les pressions de nuisance et la richesse spécifique $(r=-0,82 ; p=0,05)$. Par contre, malgré la corrélation négative observée entre les pressions de nuisance et l'abondance des espèces, elle n'est pas significative. Quant à la corrélation entre la superficie, l'abondance et la richesse spécifique, elle est positive et significative.

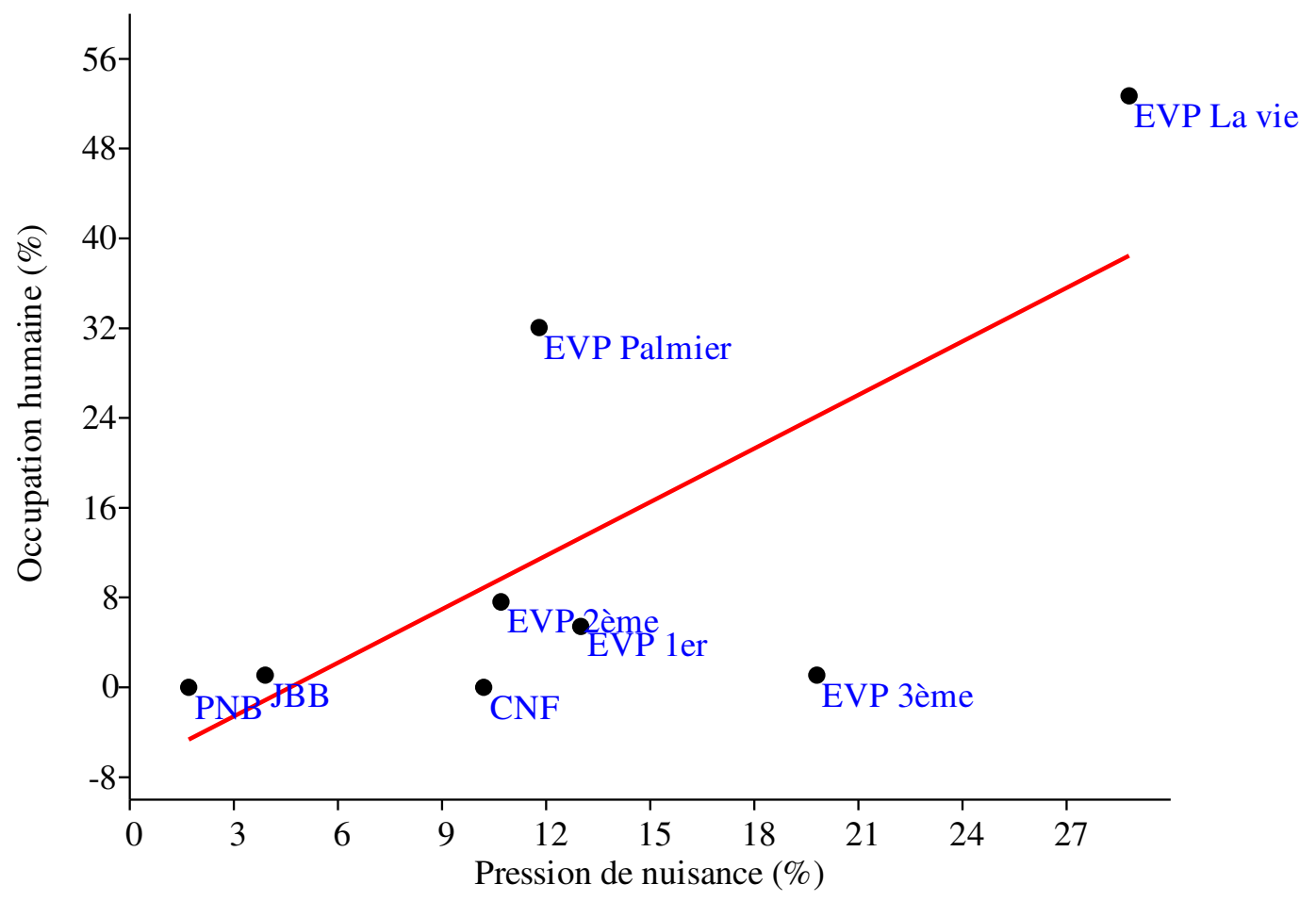

Figure 4 : Relation entre le pourcentage d'occupation humaine des espaces verts et parcs urbains et le pourcentage des pressions de nuisance $(r=0,70 ; p=0,05 ; Y=2,25 X-15,70)$. 
Kouakou et al., J. Appl. Biosci. 2018 Espaces verts comme une alternative de conservation de la biodiversité en villes : le cas des fourmis (Hyménoptère : Formicidae) dans Abidjan , Côte d'Ivoire

Tableau 6 : Corrélation de Spearman examinant la relation entre la richesse spécifique, l'abondance des fourmis et les variables relevées dans les espaces verts d'Abidjan.

\begin{tabular}{l|c|c|c|c|c|c}
\hline & $\begin{array}{c}\text { Connectivité } \\
\text { au PNB }\end{array}$ & $\begin{array}{c}\text { Pression de } \\
\text { nuisance }\end{array}$ & $\begin{array}{c}\text { Occupation } \\
\text { humaine }\end{array}$ & $\begin{array}{c}\text { Superf } \\
\text { icie }\end{array}$ & $\begin{array}{c}\text { Abondance } \\
\text { relative }\end{array}$ & $\begin{array}{c}\text { Richesse } \\
\text { spécifique }\end{array}$ \\
\hline $\begin{array}{l}\text { Distance au } \\
\text { PNB }\end{array}$ & - & 0,93487 & 0,848 & 0,664 & 0,389 & 0,664 \\
\hline $\begin{array}{l}\text { Pression de } \\
\text { nuisance }\end{array}$ & 0,05 & - & 0,068 & 0,057 & 0,326 & $\mathbf{0 , 0 5}$ \\
\hline $\begin{array}{l}\text { Occupation } \\
\text { humaine }\end{array}$ & 0,08 & 0,69 & - & 0,096 & $\mathbf{0 , 0 1 8}$ & $\mathbf{0 , 0 0}$ \\
\hline Superficie & 0,17 & $-0,71$ & $-0,64$ & - & $\mathbf{0 , 0 2 4}$ & $\mathbf{0 , 0 1 9}$ \\
\hline $\begin{array}{l}\text { Abondance } \\
\text { relative }\end{array}$ & $-0,33$ & $-0,40$ & $-\mathbf{0 , 8 2}$ & $\mathbf{0 , 4 5}$ & - & 0,022 \\
\hline $\begin{array}{l}\text { Richesse } \\
\text { spécifique }\end{array}$ & $-0,16$ & $-\mathbf{0 , 6 9}$ & $\mathbf{- 0 , 9 6}$ & $\mathbf{0 , 5 2}$ & 0,81 & - \\
\hline
\end{tabular}

Composition spécifique des fourmis, rôle de conservation et connectivité des espaces verts : De façon générale, les résultats indiquent quatre communautés distinctes de fourmis (Figure 5): la communauté du Parc National du Banco, celle regroupant le Jardin Botanique de Bingerville et le centre National de Floristique, la communauté de l'espace vert public le Palmier et celle des espaces verts publics des ponts. La composition en espèce de fourmis diffère significativement entre ces communautés (ANOSIM: $R=0,80 ; p=0,0001$ ). Toutefois, la comparaison deux à deux indique une similarité plus importante entre le Parc National du Banco et le Jardin botanique de Bingerville (42,55 \% soit 40 espèces partagées sur 94 ), suivi de la similarité entre le Parc National du Banco et le Centre National de Floristique $(34,04 \%$ soit $32 / 94)$ et de la similarité entre le Parc National du Banco et l'EVP 3ème pont $(22,34 \%$ soit 21/94). La similarité est plus faible lorsqu'on compare le Parc National du Banco à l'EVP 1er pont (17, 02 soit 16/94), à l'EVP 2ème pont (14.89\% soit 14/94), à l'EVP le Palmier (10.64 \% soit 10/94) et à l'EVP La vie" (8.51\% soit 8/94). De plus, les résultats indiquent une faible connectivité des espaces verts entre eux et au Parc National du Banco (Tableau 7). Les espaces verts les plus proches du Parc National du Banco sont l'espace vert public du 1er pont, l'espace vert public du 2ème pont et l'espace vert public du 3ème pont. 


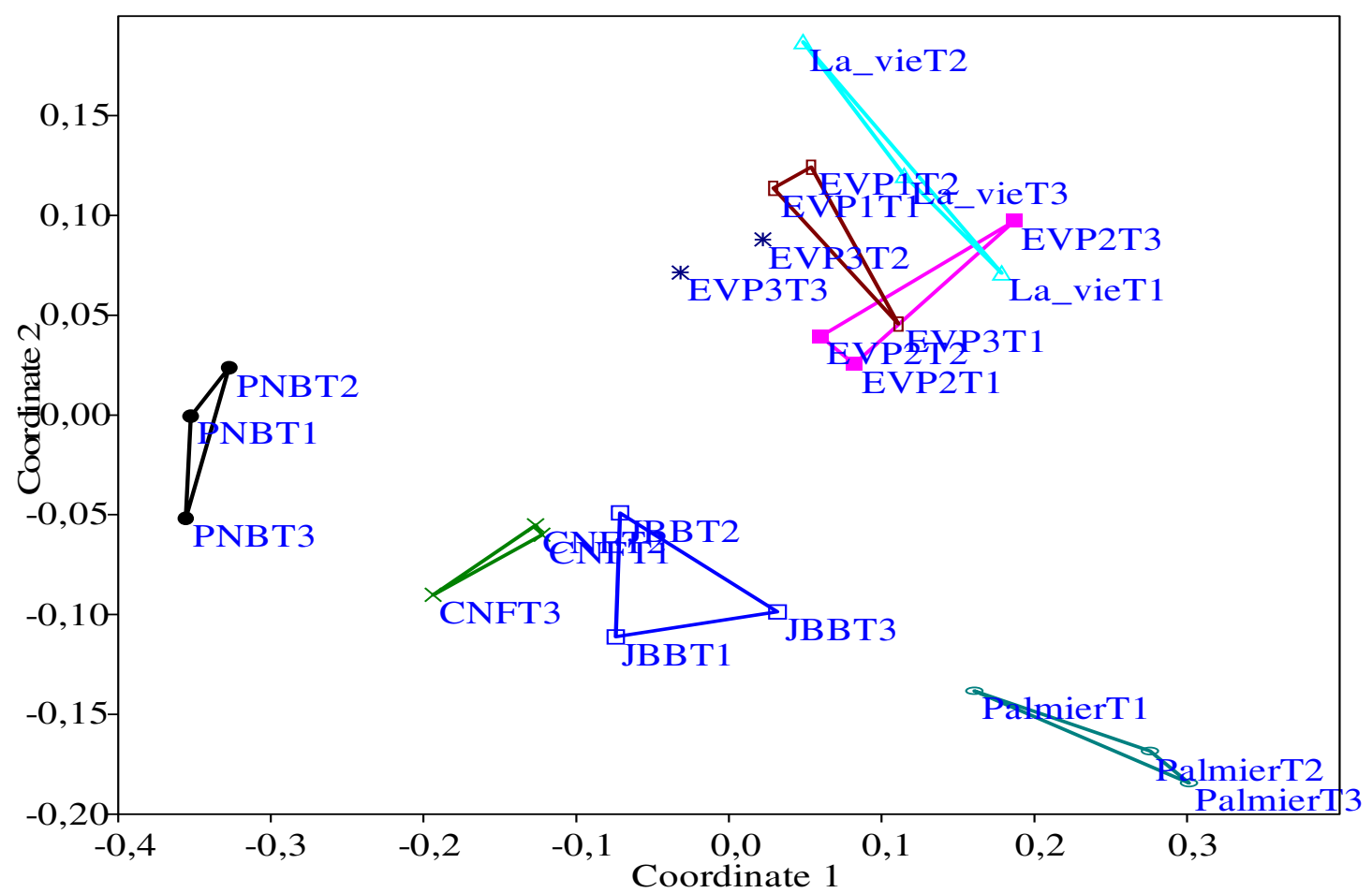

Figure 5: Graphe d'ordination de positionnement multidimensionnel (NMDS) montrant les différences dans la composition spécifique de fourmis des transects (numéroté de T1 à T3) localisés dans les espaces verts et parcs urbains (Axe $1=64,2 \% ;$ Axe $2=20 \%$; stress=0,17).

Tableau 7: Distances de connectivité en kilomètres (matrice triangulaire inférieure) et le nombre d'espèces partagées (matrice triangulaire supérieure) entre les espaces verts d'Abidjan

\begin{tabular}{l|c|c|c|c|c|c|c|c}
\hline & PNB & JBB & CNF & EVP 1 er & EVP 2 & EVP 3ème & EVP Palmier & EVP La Vie \\
\hline PNB & - & $\mathbf{2 2}$ & $\mathbf{2 1}$ & $\mathbf{1 8}$ & $\mathbf{1 8}$ & $\mathbf{2 1}$ & $\mathbf{1 3}$ & $\mathbf{8}$ \\
JBB & 12,1 & - & 23 & 23 & 22 & 20 & 21 & 20 \\
CNF & 5,9 & 10,6 & - & 14 & 12 & 19 & 16 & 15 \\
EVP 1er & 2,1 & 19,7 & 9,32 & - & 19 & 26 & 13 & 17 \\
EVP 2ème & 2,9 & 20,4 & 10 & 0,6 & - & 22 & 15 & 14 \\
EVP 3ème & 3,5 & 21,5 & 11,9 & 1,8 & 1,1 & - & 14 & 20 \\
EVP Palmier & 4,5 & 12,7 & 9,2 & 12,7 & 12,9 & 13,5 & - & 15 \\
EVP La Vie & 7,8 & 12,8 & 2,3 & 7,1 & 7,8 & 8,9 & 9,6 & - \\
\hline
\end{tabular}

\section{DISCUSSION}

Évaluation des catégories de pressions dans les espaces verts et parcs urbains: Globalement, les résultats indiquent que les espaces verts sont soumis à d'importantes pressions. Ces résultats traduiraient le fait que l'utilisation des espaces verts par la population va à l'encontre du rôle premier qui leur ait attribué, à savoir un lieu de repos et de distraction. II a été constaté que ces espaces sont illégalement occupés par des citadins, en particulier les espaces verts publics du 1er pont, du 2ème pont, du 3ème pont, La vie et le
Palmier qui constituent des lieux de dépôt d'ordures ménagères, des sites de brûlage de pneus usés, des sites horticoles, et des sites où se développent des activités commerciales. En ce qui concerne le Parc National du Banco, le Centre National de Floristique et le Jardin botanique de Bingerville, les pressions plus faibles pourraient être dues au fait que ces espaces verts sont protégés de la population. Toutefois, quelques pressions de nuisances ont été relevées. Au Centre National de Floristique, les pressions identifiées 
sont principalement les dépôts d'émondes de gazon et les déchets humains. Au Parc National du Banco, ces pressions sont dues aux visiteurs qui jettent les sachets d'eau et des pots en plastique lorsqu'ils vont rendre culte à la rivière Banco. Au Jardin botanique de Bingerville, les pressions de nuisance sont importantes à la périphérie à cause de la proximité des ménages. On a constaté au cours de l'étude, la présence de déchets humains et des ordures ménagères qui ont été jetées depuis des habitations proches de la clôture de ce jardin.

Diversité, richesse spécifique et Abondance des fourmis : Le taux de couverture de l'échantillonnage a indiqué que $74 \%$ des espèces attendues ont été collectées, signifiant que les méthodes utilisées au cours de cette étude seraient adaptées pour l'étude des communautés de fourmis des espaces verts et parcs urbains. En considérant le nombre important d'espèces de fourmis collectées (176 espèces) et le petit nombre espèces de fourmis potentiellement invasives rencontrées, nos résultats traduisent le rôle important que jouerait les espaces verts et parcs urbains comme habitats refuges pour de nombreuses espèces en milieu urbain, particulièrement les fourmis. Toutefois il est à signaler que des 6 espèces de fourmis potentiellement invasives rencontrées, Cardiocondyla emeryi, Paratrechina longicornis, Pheidole megacephala, Monomorium floricola et Tetramorium simillimum sont abondantes dans les jardins botaniques et les espaces verts publics tandis que Tapinoma melanocephalum a été la seule espèce repérée au Parc National du Banco. Cette observation s'expliquerait par le fait que ces fourmis sont des espèces vagabondes qui vivent dans les constructions humaines (Kouakou et al., 2018). Leur présence dans les espaces verts et les jardins botaniques en particulier serait due à l'introduction par les activités humaines. Cela pourrait être le cas en ce qui concerne la présence de Tapinoma melanocephalum au Banco, toutefois cette fourmi pourrait être native des forêts tropicales puisqu'elle a été collectée une seule fois.

D'une manière générale, la variation significative de la diversité spécifique, de la richesse spécifique et de l'abondance des fourmis entre les espaces verts et parcs urbains explorés suggèrerait que la typologie et la qualité de ces habitats peut influencer les communautés de fourmis qu'ils abritent. Par exemple, la richesse spécifique, la diversité spécifique et l'abondance des fourmis sont plus élevées dans le parc urbain naturel du Parc National du Banco, et modérées au Jardin botanique de Bingerville et au Centre
National de Floristique qui sont des espaces verts avec un minimum de protection. En revanche les espaces verts publics du 1er pont, du 2ème pont, l'espace vert public La vie et l'espace vert public le Palmier, accessibles à la population, présentent les plus faibles richesses spécifiques. Cette observation concorde avec les travaux faits par Uno et al. (2010), qui, au cours d'une étude menée dans trois types d'espace vert localisés dans la ville de Detroit et de Toledo aux États Unis, ont trouvé que la richesse spécifique des fourmis était élevée dans les fragments de forêts naturelles alors qu'elle était faible dans les jardins et les lots vacants.

II convient aussi de signaler qu'exceptionnellement, l'espace vert public du 3ème pont héberge un nombre important de fourmis. En fait cet espace vert est peu influencé car, bien qu'on ait noté la présence d'émondes de gazon et de déchets anthropiques, la présence humaine était relativement faible. Des résultats analogues ont été obtenus par Barbosa et al. (2015) et Graham et al. (2004) qui ont montré que le degré et le type de perturbation d'un habitat contribuaient à réduire la diversité et la richesse spécifique des fourmis. En outre, la richesse spécifique élevée pourrait être également liée à la présence de lots vacants enherbés situés entre cet espace vert et la cité de la brigade anti-émeute. Ce résultat suggèrerait que même avec une taille réduite, un espace vert public moins isolé, peu perturbé par la présence humaine et géré de façon à maximiser la biodiversité peut héberger une communauté de fourmis riche en espèces.

Influence des variables caractéristiques des espaces verts sur les paramètres des communautés: Les résultats ont révélé une forte relation entre le taux d'occupation des espaces verts et les pressions de nuisances. Ce résultat traduit le fait qu'une occupation illégale intense des espaces verts pourrait conduire à leur dégradation complète voire leur disparition. Par exemple, la forte occupation des espaces verts par les activités humaines pourrait réduire considérablement la diversité et la richesse spécifique ainsi que l'abondance des espèces natives ayant trouvé refuge dans ces milieux au profit des espèces de fourmis vagabondes plus adaptées aux perturbations anthropiques (Floren \& Linsenmair, 2001 ; Graham et al., 2004 ; McKinney, 2008 ; Basset et al., 2008). C'est le cas des espaces verts publics du $1^{\text {er }}$ pont, du $2^{\text {ème }}$ pont, l'espace vert public La vie et l'espace vert public le Palmier où l'on a noté des pressions anthropiques importantes à cause de leur occupation par les cultures horticoles, les restaurants et 
les buvettes. Quant à l'influence de la taille des espaces verts sur les communautés de fourmis, on a noté pour le Parc National du Banco, le Centre National de Floristique et le Jardin botanique de Bingerville que l'abondance et la richesse spécifique des fourmis augmente avec la taille. Alors que l'effet contraire se produit dans les espaces verts publics. Ce résultat suggèrerait qu'en plus de la taille des espaces verts, d'autres facteurs tels que les pressions anthropiques doivent être pris en compte pour expliquer la variation des paramètres de communauté de fourmis. Ces observations confortent celles d'autres chercheurs (Yamaguchi, 2004 ; Philpott et al., 2006; Fahrig et al., 2013; Barbosa et al., 2015) qui ont montré qu'en plus de la taille et de l'hétérogénéité des taches d'habitats, les perturbations liées aux activités humaines sont également des facteurs qui influencent les espèces vivant dans les habitats fragmentés. Cette tendance est démontrée lorsqu'on considère le cas particulier de l'espace vert public le Palmier. Ce site est le plus grand en taille après le PNB, le JBB et le CNF. II est également connecté aux lots vacants et à la végétation naturelle. Pourtant il présente une des plus faibles abondances et richesses de fourmis à cause du taux d'occupation et les pressions de nuisance élevées.

Composition spécifique des fourmis, rôle de conservation et connectivité des espaces verts: Les résultats indiquent une différence importante dans la composition spécifique des communautés de fourmis des espaces verts et parcs urbains. Cette différence globale pourrait s'expliquer par la faible connectivité entre les espaces verts. Selon (Lindborg et al., 2012) la connectivité des habitats est un facteur important pour la préservation de la biodiversité dans le paysage urbain. II soutient que la connectivité favorise le mouvement des espèces et permet le flux de gènes entre les espèces dans les taches d'habitat. Ainsi dans cette étude, l'isolement des espaces verts dû à l'absence de corridors écologiques affecterait la dispersion des espèces de fourmis et limiterait la recolonisation des taches d'habitats isolés. Par exemple, seuls les espaces verts du pont sont proches du PNB avec une distance minimale de 2,1 km entre

\section{CONCLUSION}

Cette étude a montré que les espaces verts et parcs urbains du district d'Abidjan ont un potentiel de conservation de la myrmécofaune native de fourmis et par conséquent, la biodiversité dans le district d'Abidjan, quelle que soit leur taille. Toutefois, les pressions de nuisances, l'occupation illégale par les l'espace vert public du $1^{\text {er }}$ pont et le PNB et une distance maximale de $3,5 \mathrm{~km}$ entre l'espace vert public du $3^{\text {ème }}$ pont et le PNB. (Haddad \& Baum, 1999) ont aussi montré que la présence de corridor favorise le déplacement des espèces de papillons entre des fragments d'habitats et par conséquent une augmentation de la densité des populations et la composition en espèce de papillons entre les fragments connectés. D'autres travaux soulignent l'importance de la connectivité entre les fragments d'habitat (Dupré et al., 2002). Ces études ont montré qu'une distance d'isolement de 1000 mètres affectait la capacité de dispersion de certains insectes, notamment les papillons. La qualité et l'état de perturbation des espaces verts pourraient également expliquer la différence dans la composition spécifique. A l'exception du Parc National du Banco, du Jardin botanique de Bingerville, du Centre National de Floristique, les autres espaces verts sont soumis à des perturbations continues à cause de la présence humaine dans ces lieux. Ces pressions pourraient avoir favorisé la prédominance des espèces de fourmis anthropophiles ou limiter les ressources alimentaires et les possibilités de nidification pour les espèces natives. A titre d'exemple, l'on a noté que le Jardin botanique de Bingerville, le Centre National de Floristique et l'espace vert public du $3^{\text {ème }}$ pont hébergeaient au moins $22 \%$ des espèces de fourmis collectées au Parc National du Banco, probablement parce que ces espaces verts sont protégés ou peu perturbés. Quant aux espaces verts du 1 er pont et l'espace vert public du $2^{\text {ème }}$ pont, ils abritent au moins $14,89 \%$ des espèces de fourmis alors que les espaces verts publics La vie et le Palmier abritent respectivement $10,64 \%$ et $8,51 \%$ de la composition en espèces du Parc National du Banco. Le pourcentage relativement important d'espèces partagées entre les espaces verts du pont et le PNB s'expliquerait par leur proximité contrairement aux espaces verts publics le Palmier et La vie qui sont influencés par l'occupation humaine en plus de leur fort éloignement au Parc National du Banco. Ce résultat traduirait la ressemblance physique ou qualitative entre certains espaces verts comme l'indique le graphe d'ordination.

activités humaines et l'absence de connectivité entre ces espaces verts et le parc urbain du Banco constituent un frein. Toutes ces pressions anthropiques empêchent ces espaces verts de jouer le rôle d'alternative pour la conservation de la biodiversité dans la ville d'Abidjan. Certains espaces verts, 
notamment le Centre National de Floristique, le Jardin botanique de Bingerville et l'espace vert du $3^{\text {ème }}$ pont à Yopougon ont montré leur capacité à préserver la biodiversité avec un minimum de connectivité et un contrôle de la pression urbaine. II peut être déduit que l'existence de taches d'habitats dans l'agglomération avec une forte connectivité et une réduction des

\section{REMERCIEMENTS}

Nous remercions le Capitaine COULIBALY Sanga, le Professeur IPOU Ipou Joseph, le Commandant COULIBALY Fousseni et l'Office Ivoirien des parcs et Réserves (OIPR) pour les autorisations qu'ils nous ont accordées pendant la réalisation de cette étude. Notre reconnaissance va également à l'endroit de SILUE Marcel, YEO Nanga, YEO Tayirou et KONAN Marius pour leur aide précieuse pendant l'étude. Nos sincères remerciements à la Fondation Internationale pour la

\section{RÉFÉRENCES BIBLIOGRAPHIQUES}

Abdourahamane IS, Amadou OA., Abdou L, Mahamane A, Saadou M, 2016. Urban Biodiversity: Perception, Preference, General Awareness, and Threats in Two Cities (Niamey and Maradi) of Niger, Urban Studies Research 2016: 12.

Agosti D, Majer JD, Alonso LE, Schultz TR, 2000. Ants: standard methods for measuring and monitoring biodiversity. Smithsonian Institution Press, Washington DC. 280 pp.

Alberti M, 2010. Maintaining ecological integrity and sustaining ecosystem function in urban areas. Current Opinion in Environmental Sustainability 2(3):178-184.

Anderson PML, Okereke C, Rudd A, Parnell S, 2013. Regional Assessment of Africa. In T. Elmqvist, M. Fragkias, J. Goodness, B. Güneralp, P. J. Marcotullio, R. I. McDonald, ... C. Wilkinson (Éd.), Urbanization, Biodiversity and Ecosystem Services: Challenges and Opportunities: A Global Assessment. Springer Netherlands, $p$ 453 459

Barbosa BC, Fagundes R, Silva LF, Tofoli JFV, Santos AM, Imai BYP, Gomes GG, Hermidoff MM, Ribeiro SP, 2015. Evidences that human disturbance simplify the ant fauna associated a Stachytarpheta glabra Cham. (Verbenaceae) compromising the benefits of ant-plant mutualism. Revista Brasleira De Biologia 75(1): 58-68. pressions anthropiques pourraient permettre de maintenir les communautés de fourmis natives et par ricochet la biodiversité en ville. Enfin, les résultats ont montré également l'importance des fourmis comme outils de bio-monitoring pour l'évaluation de l'état de santé des fragments d'habitats du milieu urbain.

Science (IFS) qui a accordé à Monsieur KOUAKOU Lombart Mesmer Maurice la bourse $N^{\circ} \mathrm{D} / 5837-1$ qui a permis de collecter les données au cours de cette étude. Nous remercions également le Point Focale Belge pour l'Initiative Taxonomique Mondiale (GTI) et I'Institut Royal des Sciences Naturelles de Belgique pour la bourse $\mathrm{N}^{\circ} \mathrm{CBD} / \mathrm{GTI}-02 / \mathrm{MLS} / 2017.27$ qu'ils nous ont octroyé pour l'identification des fourmis.

Basset Y, Missa O, Alonso A, Miller SE, Curletti G, De Meyer $M$, ...Wagner $T, 2008$. Changes in arthropod assemblages along a wide gradient of disturbance in Gabon. Conservation Biology: The Journal of the Society for Conservation Biology 22(6): 1552-1563.

Bharti $H$, Bharti $M$, Pfeiffer $M, 2016$. Ants as bioindicators of ecosystem health in Shivalik Mountains of Himalayas: assessment of species diversity and invasive species. Asian Myrmecology 8: 1-15

Bolton B, 1976. The ant tribe Tetramoriini (Hymenoptera: Formicidae). Constituent genera, review of smaller genera and revision of Triglyphothrix Forel. Bulletin of the British Museum (Natural History) 34: 283-379.

Bolton B, 1980. The ant tribe Tetramoriini (Hymenoptera: Formicidae). The genus Tetramorium Mayr in the Ethiopian zoogeographical region. Bulletin of the British Museum (Natural History) 40: 193-384.

Bolton B, 1994. Identification Guide to the Ant Genera of the World - Barry Bolton | Harvard University Press. 225p.

Chapin III FSC, Zavaleta ES, Eviner VT, Naylor RL, Vitousek PM, Reynolds HL, ... Díaz S, 2000. Consequences of changing biodiversity. Nature 405: 234-242.

Del Toro I, Ribbons RR, Pellini SL, 2012. The little things that run the world revisited: a review of ant-mediated ecosystem services and 
Kouakou et al., J. Appl. Biosci. 2018 Espaces verts comme une alternative de conservation de la biodiversité en villes : le cas des fourmis (Hyménoptère : Formicidae) dans Abidjan , Côte d'Ivoire

disservices (Hymenoptera: Formicidae). Myrmecological News 17: 133-146.

Djibril K, Coulibaly A, Wang X, Ousmane D, 2012. Evaluating green space use and management in

Abidjan City, Cote d'Ivoire. International Journal of Economics and Management Engineering 2 (3): 108-116.

Dupré C. and Ehrlén J, 2002. Habitat configuration, species traits and plant distributions. Journal of Ecology 90(5):796-805.

Fahrig L. and Triantis K, 2013. Rethinking patch size and isolation effects: the habitat amount hypothesis. Journal of Biogeography 40(9): 1649-1663.

Ferland A, 2015. La conservation de la biodiversité en milieu urbain : comment aménager les villes du monde? (Essai). Université de Sherbrooke, $105 p$.

Floren A. and Linsenmair KE, 2001. The influence of anthropogenic disturbances on the structure of arboreal arthropod communities. Plant Ecology 153(102): 153-167.

Goddard MA, Dougill AJ, Benton TG, 2010. Scaling up from gardens: biodiversity conservation in urban environments. Trends in Ecology \& Evolution 25(2):90-98.

Graham JH, Hughie HH, Jones S, Wrinn K, Krzysik AJ, Duda JJ, ... Balbach H, 2004. Habitat disturbance and the diversity and abundance of ants (Formicidae) in the Southeastern FallLine Sandhills. Journal of Insect Science 4: 30

Grunewald K, Richter B, Meinel G, Herold H, Syrbe RU, 2017. Proposal of indicators regarding the provision and accessibility of green spaces for assessing the ecosystem service "recreation in the city" in Germany. International Journal of Biodiversity Science, Ecosystem Services \& Management 13(2): 26-39.

Haddad NM. and Baum KA, 1999. An Experimental Test of Corridor Effects on Butterfly Densities. Ecological Applications 9(2): 623-633.

Hoffmann BD and Andersen AN, 2003. Responses of ants to disturbance in Australia, with particular reference to functional groups. Austral Ecology 28(4): 444-464.

Hölldobler B. and Wilson EO, 1990. The Ants. Cambridge Mass, 762p.

Holway DA, Lach L, Suarez AV, Tsutsui ND, Case TJ, 2002. The causes and consequences of ant invasions. Annual Review of Ecology and Systematics 33: 181-233.

Jamison SL, Robertson M, Engelbrecht I, Hawkes P, 2016. An assessment of rehabilitation success in an African grassland using ants as bioindicators. Koedoe 58(1): 16.

Jennings V, Larson L, Yun J, 2016. Advancing Sustainability through Urban Green Space: Cultural Ecosystem Services, Equity, and Social Determinants of Health. International Journal of Environmental Research and Public Health 13(2): 196.

Kark S, Iwaniuk A, Schalimtzek A, Banker E, 2006. Living in the city: can anyone become an 'urban exploiter'? Journal of Biogeography 34(4): 638-651.

Kaspari M, 1996. Worker size and seed size selection by harvester ants in a neotropical forest. Oecologia 105(3): 397-404.

King JR. and Porter SD, 2005. Evaluation of Sampling Methods and Species Richness Estimators for Ants in Upland Ecosystems in Florida. Environmental Entomology 34(6): 156-1578.

Kone M, 2013. Effets de l'intensification des modes d'utilisation des terres et du gradient d'âge de la cacaoculture sur la diversité biologique des fourmis en zones préforestière (lamto) et forestière semidécidue (Oumé) de Côte d'Ivoire. Thèse de l'Université Félix Houphouët Boigny.177 p.

Kone M, Konate S, Yeo K, Kouassi PK and Linsenmair $\mathrm{KE}, 2014$. Effects of management intensity on ant diversity in cocoa plantation (Oume, centre west Côte d'lvoire). Journal of Insect Conservation 18(4): 701-712.

Kouakou LMM, Yeo K, Ouattara K, Dekoninck W, Delsinne T, Konaté S, 2018. Investigating urban ant community (Hymenoptera: Formicidae) in port cities and in major towns along the border in Côte d'Ivoire: a rapid assessment to detect potential introduced invasive ant species. Journal of Animal \& Plant Sciences 36 (1): 5793-5811

Kowarik I, 2011. Novel urban ecosystems, biodiversity, and conservation. Environmental Pollution 159(8): 1974-1983.

Kühn I, Brand R, Klotz, S, 2004. The flora of German cities is naturally species rich. Evolutionary Ecology Research 6(5): 749-764.

Lawton JH, Bignell DE, Bolton B, Bloemers GF, Eggleton P, Hammond PM, Watt AD, 1998. 
Biodiversity Inventories, Indicator Taxa and Effects of Habitat Modification in Tropical Forest. Nature 391: 72-76.

Lindborg R, Helm A, Bommarco R, Heikkinen RK, Kühn I, Pykälä J, Pärtel M, 2012. Effect of habitat area and isolation on plant trait distribution in European forests and grasslands. Ecography 35(4): 356-363.

Luniak M, 1994, The development of bird communities in new housing estates in Warsaw. Memorabilia Zoologica 49: 257-267

Marzluff JM, 2005. Island biogeography for an urbanizing world: how extinction and colonization may determine biological diversity in human-dominated landscapes. Urban Ecosystems 8(2): 157-177.

Mata L, Threlfall CG, Williams NSG, Hahs AK, Malipatil M, Stork NE, Livesley SJ, 2017. Conserving herbivorous and predatory insects in urban green spaces. Scientific Reports 7: 40970.

McDonnell MJ, and Hahs AK, 2013. The future of urban biodiversity research: Moving beyond the "lowhanging fruit'. Urban Ecosystems 16(3): $397 \square 409$.

McGlynn TP, 1999. The worldwide transfer of ants: geographical distribution and ecological invasions. Journal of Biogeography 26(3): 535548.

McKinney ML 2006. Urbanization as a major cause of biotic homogenization. Biological Conservation 127(3): $247 \llbracket 260$.

McKinney ML, 2008. Effects of urbanization on species richness: A review of plants and animals. Urban Ecosystems 11(2): 161-176.

Müller N, 2010. Most Frequently Occurring Vascular Plants and the Role of Non-Native Species in Urban Areas - A Comparison of Selected Cities in the Old and the New Worlds. In N. Müller PW. and Kelcey JG (Éd.), Urban Biodiversity and Design. Wiley-Blackwell, $p$ 227-242

Nassa DDA, 2009. Crise de la nature dans l'agglomération abidjanaise: l'exemple de la colonisation des espaces verts par l'habitat et les commerces dans la commune de Cocody. Ville et Organisation de l'espace en Afrique p149-158.

Oliver I. and Beattie AJ, 1996. Designing a Cost-Effective Invertebrate Survey: A Test of
Methods for Rapid Assessment of Biodiversity. Ecological Applications 6(2): 594-607.

Philpott SM, Perfecto I, Vandermeer J, 2006. Effects of Management Intensity and Season on Arboreal Ant Diversity and Abundance in Coffee Agroecosystems. Biodiversity \& Conservation 15(1): 139-155.

Ramade F, 1993. Dictionnaire encyclopédique de l'écologie et des sciences de l'environnement. Paris: Ediscience international. 760p.

Stadler B. and Dixon A, 2005. Ecology and Evolution of Aphid-Ant Interactions. Annual Review of. Ecology, Evolution and Systematics 20: 345372.

Uno S, Cotton J, Philpott SM, 2010. Diversity, abundance, and species composition of ants in urban green spaces. Urban Ecosystems 13(4): 425-441.

Wetterer JK, 2015. Geographic origin and spread of cosmopolitan ants (Hymenoptera: Formicidae). Haltères 6: 66-78.

Yamaguchi T, 2004. Influence of urbanization on ant distribution in parks of Tokyo and Chiba City, Japan I. Analysis of ant species richness. Ecological Research 19(2): 209-216.

Yeo K, Delsinne T, Konate S, Alonso LL, Aïdara D, Peeters C, 2016. Diversity and distribution of ant assemblages above and below ground in a West African forest-savannah mosaic (Lamto, Côte d'Ivoire). Insectes Sociaux 1(64): $155 \llbracket 168$.

Yeo K, Tiho S, Ouattara K, Konate S, Kouakou LMM, Fofana M, 2013. Impact de la fragmentation et de la pression humaine sur la relique forestière de l'Université d'Abobo-Adjamé (Côte d'Ivoire). Journal of Applied Biosciences 61: 45514565.

Yeo K, 2006. Dynamique spatiale et diversité des fourmis de la litière et du sol dans une mosaïque forêt-savane en Côte d'Ivoire. Thèse unique de Doctorat des universités Pierre et Marie Curie Paris VI et AboboAdjamé, $212 p$.

Yeo K, Konate S, Tiho S, Camara S K, 2011. Impacts of land use types on ant communities in a tropical forest margin (Oum Cte dlvoire). African Journal of Agricultural Research 6(1): $260 \square 274$. 